\title{
Non parametric Measures of Stability Compared as per BLUP and BLUE of Wheat Genotypes Evaluated in North Western Plains Zone of the Country
}

\author{
Ajay Verma* and G.P. Singh \\ ICAR-Indian Institute of Wheat \& Barley Research, Post Bag \# 158 Agrasain Marg, \\ Karnal 132001 (Haryana), India \\ *Corresponding author
}

\section{Keywords \\ BLUP, BLUE, $S_{i}^{(s)}$, $\mathrm{CS}_{\mathrm{i}}^{(\mathrm{s})}, \mathrm{NP}_{\mathrm{i}}^{(\mathrm{s})}$, \\ Coefficient of concordance, Biplot analysis}

\section{Article Info}

Accepted:

11 June 2020

Available Online:

10 July 2020

\section{A B S T R A C T}

Non parametric measures as per the BLUP values of original yield for first year, $S_{i}^{s}$ measures identified G11, G10, G4 as stable genotypes. Corrected yield measures $\mathrm{CS}_{i}^{\mathrm{s}}$ selected G11, G12, G8 for stable performance. Values of $\mathrm{NP}_{\mathrm{i}}{ }^{(\mathrm{s})}$ identified G4, G10, G11 as desirable genotypes. Association analysis among measures observed positive correlations of $S_{i}^{s}$, with others and of negative with CMR, Z1 and Z2. Positive relationships exhibited by $\mathrm{CS}_{\mathrm{i}}^{\mathrm{s}}$ measures. Negative relationships of $\mathrm{NP}_{\mathrm{i}}{ }^{(\mathrm{s})}$ expressed with $\mathrm{Z} 1$ and $\mathrm{Z} 2$ only. Biplot analysis showed CMed\& CMR expressed close affinity with $\mathrm{SD}, \mathrm{NP}_{\mathrm{i}}^{(3)}, \mathrm{NP}_{\mathrm{i}}{ }^{(4)}$ \& $\mathrm{S}_{i}^{\mathrm{s}}$ whereas $\mathrm{CCV}, \mathrm{CSD}, \mathrm{NP}_{\mathrm{i}}^{(1)}$ associated with $\mathrm{CS}_{\mathrm{i}}^{\mathrm{s}}$ along with $\mathrm{Z} 1$. Based on BLUE's of genotypes yield, measures $\mathrm{S}_{\mathrm{i}}^{\mathrm{s}}$ foundG4, G8, G12as suitable genotypes. $\mathrm{CS}_{\mathrm{i}}^{\mathrm{s}}$ identified G4, G8, G11, G12, as opposed to G4, G5, G8, G11by measures $\mathrm{NP}_{\mathrm{i}}{ }^{(\mathrm{s})}$ measures. Positive correlations exhibited by $\mathrm{S}_{\mathrm{i}}^{\mathrm{s}}$ except of negative with $\mathrm{CMR}, \mathrm{Z} 1$ and $\mathrm{Z} 2$. Measures $\mathrm{CS}_{\mathrm{i}}^{\mathrm{s}}$ expressed highly significant indirect relationship with $\mathrm{Z} 2$ only. Negative relationships of $\mathrm{NP}_{\mathrm{i}}^{\left({ }^{(\mathrm{s})}\right.}$ had observed with $\mathrm{Z} 2$ only. CV expressed affinity with CMR, $\mathrm{S}_{\mathrm{i}}{ }^{3}, \mathrm{~S}_{\mathrm{i}}{ }^{6}, \mathrm{NP}_{\mathrm{i}}{ }^{(2)}, \mathrm{NP}_{\mathrm{i}}{ }^{(3)}$ \& $\mathrm{NP}_{\mathrm{i}}{ }^{(4)}$ whereas SD grouped with $\mathrm{S}_{\mathrm{i}}{ }^{1}, \mathrm{~S}_{\mathrm{i}}{ }^{2}, \mathrm{~S}_{\mathrm{i}}{ }^{4}, \mathrm{~S}_{\mathrm{i}}{ }^{5}, \mathrm{~S}_{\mathrm{i}}{ }^{7}$ measures. BLUP's of genotypes for second year of study seen, measures $S_{i}{ }^{s}$ settled forG1, G9, G13, G15 genotypes. While $\mathrm{NPi}^{(1)}$ identified G9, G11,G14 and G15genotypes of stable performance. Measures CMR and $\mathrm{Z} 2$ showed negative correlations with $\mathrm{S}_{\mathrm{i}}^{\mathrm{s}}$, and $\mathrm{CS}_{\mathrm{i}}^{\mathrm{s}}$, behaved in direct manner along with indirect to $\mathrm{Z} 2$.Negative relationships of $\mathrm{NP}_{\mathrm{i}}^{(\mathrm{s})}$ exhibited with $\mathrm{Z1}$ and $\mathrm{Z2}$. $\mathrm{CV}$ along with CMR expressed affinity with, $\mathrm{SD}, \mathrm{CMed}, \mathrm{NP}_{\mathrm{i}}{ }^{(2)}, \mathrm{NP}_{\mathrm{i}}{ }^{(3)}, \mathrm{NP}_{\mathrm{i}}{ }^{(4)}, \mathrm{S}_{\mathrm{i}}{ }^{3}, \mathrm{~S}_{\mathrm{i}}{ }^{5}, \mathrm{~S}_{\mathrm{i}}{ }^{6}$. Large cluster comprises of CCV, CSD, $\mathrm{NP}_{\mathrm{i}}{ }^{(1)}, \mathrm{S}_{\mathrm{i}}{ }^{1}, \mathrm{~S}_{\mathrm{i}}{ }^{2}, \mathrm{~S}_{\mathrm{i}}{ }^{4}, \mathrm{CS}_{\mathrm{i}}{ }^{1}, \mathrm{CS}_{\mathrm{i}}{ }^{2}, \mathrm{CS}_{\mathrm{i}}{ }^{3}, \mathrm{CS}_{\mathrm{i}}{ }^{4}, \mathrm{CS}_{\mathrm{i}}{ }^{5}, \mathrm{CS}_{\mathrm{i}}{ }^{6}, \mathrm{CS}_{\mathrm{i}}{ }^{7}$ and $\mathrm{Z} 1$ measures. $\mathrm{S}_{\mathrm{i}}^{\mathrm{S}}$ non parametric while considering BLUE's values of genotypes pointed towards G5, G9, G14 whereas G6,G9 by $\mathrm{CS}_{\mathrm{i}}^{\mathrm{s}}$ values. Wheat genotypes G5,G9, G14,G15 settled by least values of $\mathrm{NP}_{\mathrm{i}}^{(\mathrm{s})}$. Indirect relations with Z2 only exhibited by $\mathrm{S}_{\mathrm{i}}^{\mathrm{s}}$ measures. $\mathrm{CS}_{\mathrm{i}}^{\mathrm{s}}$ maintained positive relationships. $\mathrm{NP}_{\mathrm{i}}{ }^{(\mathrm{s})}$ had positive relationships with others and negative relationships with $\mathrm{Z} 2$ only. Largest cluster consists of SD, CSD, CCV, $\mathrm{NP}_{\mathrm{i}}{ }^{(1)}, \mathrm{S}_{\mathrm{i}}{ }^{1}, \mathrm{~S}_{\mathrm{i}}{ }^{4}, \mathrm{~S}_{\mathrm{i}}^{5}, \mathrm{~S}_{\mathrm{i}}{ }^{7}, \mathrm{CS}_{\mathrm{i}}{ }^{1}, \mathrm{CS}_{\mathrm{i}}{ }^{2}, \mathrm{CS}_{\mathrm{i}}^{3}{ }^{3} \mathrm{CS}_{\mathrm{i}}{ }^{4}, \mathrm{CS}_{\mathrm{i}}^{5}{ }^{5} \mathrm{CS}_{\mathrm{i}}{ }^{6}, \mathrm{CS}_{\mathrm{i}}{ }^{7}$ measures. 


\section{Introduction}

Mostly genotypes possessed high yield with broad stability were recommended for diverse environmental conditions of the country to sustain the production levels of cereal crops (Pour et al., 2019). High yield stability proved the genotype's ability to perform consistently, whether at high or low-yield levels, across a wide range of environments (Vaezi et al., 2018). With weather vagaries the significant genotype-by-environment $(\mathrm{GxE})$ interaction affected the genotypes performance (Rasoli et al., 2015). Modeling GxE interaction in multienvironment trials (MET) helps to determine phenotypic stability of genotypes astwo concepts of interaction i.e., crossover, and non-crossover, have been cited in literature (Ahmadi et al., 2015). Change in the rankings of the genotypes in different environments proved the presence of crossover interaction. More over the similar rankings in various environments had been analysed by simple process with absence of crossover interaction (Zali et al., 2011). Some genotypes have similar performance regardless of the productivity level of the environment, and others have their performance directly related to the productivity potential of the environment, highlighted the importance of stability analysis (Mahtabi et al., 2013). Genotype $\mathrm{x}$ environment interactions complicate the identification of superior genotypes (Mortazavian and Azizinia, 2014) but their interpretation can be facilitated by the use of several statistical modeling methods. Two major approaches for the stability analysis of genotypes had been mentioned (Khalili et al., 2016; Mohammadi et al., 2016). First, most commonly used, approach known as parametric which presumed certain assumptions about distributions of genotypic, environmental, and their linear interaction effects. Other approach called non-parametric which had no compulsions of any assumptions. Non- parametric procedures proposed based on the ranks of genotypes in each environment, and the genotypes with similar ranking across environments were classified as stable (Farshadfar et al., 2014). Non-parametric methods had certain advantages over counterpart parametric stability methods. Reduced the bias due to outliers, if any and easy to use along with simple to interpret, and little effect on the results had been observed with additions or deletions of few genotypes (Vaezi et al., 2018).Ranking of genotypes had classified genotypes according to values but not to their absolute differences. However, nonparametric procedures are used less often then parametric procedures despite certain advantages (Mohammadi et al., 2016). Quite large number of nonparametric procedures has been developed to interpret stability in GxE interaction studies to determine whether or not genotypes evaluated in MET were possessing stable yield performance (Delić et al., 2009; Kilic et al., 2010; Balalić et al., 2011; Karimizadeh et al., 2012; Mortazavian and Azizinia 2014; Pour et al., 2019).

The present study was carried out with the objectives (1) analyze stability analysis genotypes by nonparametric measures using BLUP and BLUE values (2) to differentiate the yield and stable patternof genotypes vis-àvis BLUP and BLUE and (3) study the relationships, similarities and dissimilarities among non-parametric measures of stability.

\section{Materials and Methods}

Twelve promising wheat genotypes were evaluated in research field trials at 28 centers of All India Coordinated Research Project on Wheat across mega zone of the country during 2016-17 and fifteen at twenty-five centers during 2017-18 cropping season. Field trials were laid out in Randomized block designs with fore replications. Recommended practices of packages had followed in total to 
harvest the good yield. Parentage details and environmental conditions were reflected in tables $1 \& 2$ for ready reference. Huehn (1990 a \&b) proposed seven nonparametric methods for assessing GxE interaction and stability analysis. For a two-way dataset with $\mathrm{k}$ genotypes and $n$ environments $\mathrm{X}_{\mathrm{ij}}$ denotes the phenotypic value of ith genotype in $\mathrm{jth}$ environment where $i=1,2, \ldots k, j=, 1,2, \ldots, n$ and $r_{i j}$ as the rank of the ith genotype in the $j$ th environment, and $\overline{r_{i}}$ as the mean rank across all environments for the ith genotype. Sabaghnia et al., (2012) proposed the correction for yield of ith genotype in jth environment as $\left(\mathrm{X}_{\mathrm{ij}}=\mathrm{X}_{\mathrm{ij}}{\overline{x_{\mathrm{u}}}} \overline{\bar{x}}_{\mathrm{m}}\right)$ as $\mathrm{X}_{\mathrm{ij}}$, was the corrected phenotypic value; $\bar{X}_{1}$.was the mean of ith genotype in all environments and $\bar{X}_{m}$ was the grand mean. Generally used seven statistics based on ranks of genotypes yield and corrected yield were expressed as follows:

$N P_{i}^{(1)}=\frac{1}{n} \sum_{j=1}^{n} \| r_{i j}^{*}-M_{d i}^{*} \mid$

$N P_{i}^{(2)}=\frac{1}{n}\left(\frac{\sum_{j=1}^{n}\left\|r_{i j}^{*}-M_{d i}^{8}\right\|}{M_{d i}}\right)$

$$
\begin{aligned}
& N P_{i}^{(3)}=\frac{\sqrt{\sum\left(r_{i j}^{*}-\bar{r}_{i,}^{*}\right)^{2} / n}}{\bar{r}_{i *}} \\
& N P_{i}^{(4)}=\frac{2}{n(n-1)}\left[\sum_{j=1}^{n-1} \Sigma_{j^{\prime}=j+1}^{m} \frac{\left.\left|r_{i j}^{*}-r_{i j}^{*}\right|\right]}{\bar{r}_{i,}^{*}}\right]
\end{aligned}
$$

Significance of $\mathrm{Si}^{(1)}$ and $\mathrm{Si}^{(2)}$ non parametric measures had been explored by Nassar and Huehn (1987). Z1 and Z2 values were calculated for each genotype, based on the ranks of adjusted data and then sum of i.e. $Z_{1}$ sum and $Z_{2}$ sum are distributed as $\chi^{2}$. Degree of similarity among measures had assessed by estimating correlation coefficients while considering genotypes ranking. Spearman's rank correlation values among pairs (Piepho and Lotito, 1992) estimated as follows:

$\bar{r}_{s}=1-\frac{6 \sum_{i=1}^{n} d_{i}^{2}}{n\left(n^{2}-1\right)}$ where $d_{i}$ denotes difference between ranks for ith genotype and $n$ is total number of pairs.

\section{Results and Discussion}

\section{First year of study 2016-17}

\section{Analytic analysis as per BLUP's} followed by G7 and G6, although differences were evident among the genotypes (Table 3). mean of ranks (MR), standard deviation of

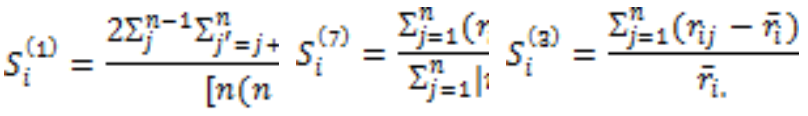

$$
\begin{aligned}
& s_{i}^{(4)}=\sqrt{\frac{\sum_{j=1}^{n}\left(r_{i j}-\right.}{n}} S_{i}^{(5)}=\frac{\sum_{j=1}^{n} \mid \eta_{i}}{n} s_{i}^{(\omega)}=\frac{\sum_{j=1}^{n} \mid n_{i j}-\bar{r}_{i}}{\bar{r}_{i,}}
\end{aligned}
$$

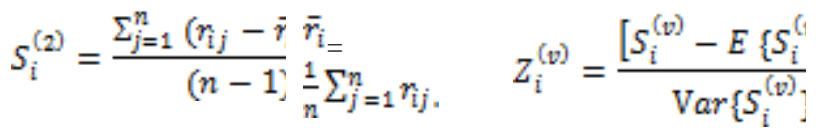

Non parametric measures for stability analysis proposed by Thennarasu (1995) as $\mathrm{NP}_{\mathrm{i}}{ }^{(1)}$, $\mathrm{NP}_{\mathrm{i}}{ }^{(2)}, \mathrm{NP}_{\mathrm{i}}{ }^{(3)}$ and $\mathrm{NP}_{\mathrm{i}}^{(4)}$ based on ranks of corrected means of genotypes. In the formulas, $\mathrm{r}_{\mathrm{ij}}^{*}$ was the rank of $\mathrm{X}_{\mathrm{ij}}^{*}$, and $\bar{r}_{\mathrm{i}}$ and $\mathrm{M}_{\mathrm{di}}$ were the mean and median ranks for original (unadjusted) grain yield, where $\bar{r}_{z}^{*}$ and $\mathrm{M}_{\mathrm{di}}^{*}$ were the same parameters computed from the corrected (adjusted) data.

Average yield of wheat genotypes observed G1 was the highest yielding with $57.4 \mathrm{q} / \mathrm{ha}$ Measure GAI selected G1, G12, G7 as genotypes with higher adaptable index values. The following three descriptive statistics; ranks (SD) and coefficient of variation of ranks $(\mathrm{CV})$ were calculated as per original 
values of genotypes yield. MR pointed towards G5, G4, G3 and SD for G11,G8, G10 whereas CV for G11,G4, G10 as genotypes of stable performance, while G5, G3 based on MR;G1, G5 based on SD and G6, G1 based on $\mathrm{CV}$, would be genotypes of unstable behavior. These descriptive statistics based on ranks can be used for genotype comparative evaluation. Two ranking methods according to mean and standard deviation of ranks proposed by Sabaghnia et al., (2012) and advantages of non - parametric procedures in phenotypic stability studies had reported by Rasoli et al., (2015). Median selected G6, G7, G9 wheat genotypes. Seven nonparametric measures based on original yield of genotypes $\left(\mathrm{S}_{\mathrm{i}}{ }^{1}, \quad \mathrm{~S}_{\mathrm{i}}{ }^{2}, \quad \mathrm{~S}_{\mathrm{i}}{ }^{3}, \quad \mathrm{~S}_{\mathrm{i}}{ }^{4}, \quad \mathrm{~S}_{\mathrm{i}}{ }^{5}, \quad \mathrm{~S}_{\mathrm{i}}{ }^{6}, \quad \mathrm{~S}_{\mathrm{i}}{ }^{7}\right)$ indicated G11,G10,G4 as the stable genotypes, however G1, G6, would achieve unstable yield. According to corrected yield values (table 4), G1,G8 \& G10 were of choice by mean of corrected ranks (CMR), G12,G11 \&G8 by standard deviation of corrected ranks (CSD) and G8,G10 \& G4 were of stable nature as per coefficient of variation of corrected ranks (CCV). Measures based on corrected yield $\left(\mathrm{CS}_{\mathrm{i}}{ }^{1}, \mathrm{CS}_{\mathrm{i}}{ }^{2}, \mathrm{CS}_{\mathrm{i}}{ }^{3}, \mathrm{CS}_{\mathrm{i}}{ }^{4}, \mathrm{CS}_{\mathrm{i}}{ }^{5}, \mathrm{CS}_{\mathrm{i}}{ }^{6}, \mathrm{CS}_{\mathrm{i}}{ }^{7}\right)$ identified G11, G12, G8 as stable genotypes and G5, G1 would be of unstable type. The mentioned strategy determines the stability of genotype over environment if its rank is similar over other environments (biological concept). Many authors that have used the corrected Huehn's (1979, 1990b) nonparametric measures of phenotypic stability and demonstrated that these statistics were associated with the biological concept of stability (Karimizadeh et al., 2012; Sabaghnia et al., 2012; Ahmadi et al., 2015). Results of Thennarasu's (1995) non-parametric measures, which considered the ranks of adjusted yield means, were shown in Table 4 . As per values of first measure $\mathrm{NP}_{\mathrm{i}}^{(1)}, \mathrm{G} 11$, G10 and G12 genotypes were considered as of stable type in comparison to the other genotypes. The genotypes G11, G4 and G10 had the lower value of $\mathrm{NP}_{\mathrm{i}}^{(2)}, \mathrm{NP}_{\mathrm{i}}{ }^{(3)}$ considered G4, G11 followed by G10, whereas $\mathrm{NP}_{\mathrm{i}}{ }^{(4)}$ identified $\mathrm{G} 4, \mathrm{G} 11$ and G10. The most unstable genotypes based on $\mathrm{NP}_{\mathrm{i}}{ }^{(\mathrm{s})}$ were G6 followed by G1 and G5. MeasuresZ1 and Z2 were the standardized values $\mathrm{CS}_{\mathrm{i}}{ }^{1} \& \mathrm{CS}_{\mathrm{i}}{ }^{2}$ had pointed for G9, G2, G7 genotypes.

To judge the degree of agreement among nonparametric measures, Kendall's coefficient of concordance, W was used. Numerical values equal to 1 indicates a perfect agreement among rankings of the measures across the environments and near to 0 suggests total disagreement among ranks (Vaezi et al., 2018).Calculated values of $\mathrm{W}$ and significance of numerical value judged by $\chi^{2}$ statistic were given in Table 4. Since calculated value is less than table of $\chi^{2}(0.05$, $290)=124.3$ (135.8), resulted an overall similarity among non parametric measures. Significance of $\mathrm{CS}_{\mathrm{i}}{ }^{1}$ and $\mathrm{CS}_{\mathrm{i}}{ }^{2}$ were tested as per Nassar and Huehn (1987). For each genotype, Z1 and Z2 values were calculated based on the ranks of adjusted data and then summed: $Z_{1}$ sum $=30.37$ and $Z_{2}$ sum $=41.63$ (Table 4). Both these statistics are distributed as $\chi^{2}$ and were less than the critical value of $\chi^{2}(0.05,29)=42.6$. This indicated the nonsignificant differences among genotypes as per ranks of $\mathrm{CS}_{\mathrm{i}}{ }^{1}$ and $\mathrm{CS}_{\mathrm{i}}{ }^{2}$ measures. More over the individual $\mathrm{Z}$ values showed BRW3773\&HD2967 were significantly unstable relative to others, with their individual $\mathrm{Z}$ values more than the critical value of $\chi^{2}(0.05,1)=3.84$.

\section{Association analysis}

Spearman's rank correlation analysis among possible pairs of non parametric measures (Table 11) observed highly significant $(\mathrm{p}<$ 0.01 ) positive correlation of yield with GAI, Med and negative correlation with $\mathrm{MR}, \mathrm{CV}$, $\mathrm{S}_{\mathrm{i}}{ }^{6}, \mathrm{NP}_{\mathrm{i}}{ }^{(2)}, \mathrm{NP}_{\mathrm{i}}{ }^{(3)}, \mathrm{NP}_{\mathrm{i}}{ }^{(4)}$ along with indirect relationships with $\mathrm{SD}, \mathrm{S}_{\mathrm{i}}{ }^{1}, \mathrm{~S}_{\mathrm{i}}{ }^{2}, \mathrm{~S}_{\mathrm{i}}{ }^{3}, \mathrm{~S}_{\mathrm{i}}{ }^{4}, \mathrm{~S}_{\mathrm{i}}{ }^{5}, \mathrm{~S}_{\mathrm{i}}{ }^{7}$, 
$\mathrm{CS}_{\mathrm{i}}{ }^{1}, \mathrm{CS}_{\mathrm{i}}{ }^{2}, \mathrm{CS}_{\mathrm{i}}{ }^{3}, \mathrm{CS}_{\mathrm{i}}{ }^{4}, \mathrm{CS}_{\mathrm{i}}{ }^{5}, \mathrm{CS}_{\mathrm{i}}{ }^{6}$ and $\mathrm{CS}_{\mathrm{i}}{ }^{7}$. GAI measure showed negative correlations with $\mathrm{MR}, \mathrm{CV}, \mathrm{S}_{\mathrm{i}}{ }^{6}, \mathrm{NP}_{\mathrm{i}}{ }^{(2)}, \mathrm{NP}_{\mathrm{i}}{ }^{(3)}, \mathrm{NP}_{\mathrm{i}}{ }^{(4)}$ and positive with median. Mean rank (MR) expressed positive correlation with $\mathrm{CV}, \mathrm{S}_{\mathrm{i}}^{3}, \mathrm{~S}_{\mathrm{i}}{ }^{6}, \mathrm{NP}_{\mathrm{i}}{ }^{(2)}$, $\mathrm{NP}_{\mathrm{i}}^{(3)}, \mathrm{NP}_{\mathrm{i}}^{(4)}$ and negative with Med. Measure SD maintained highly significant and significant direct relations with almost all the measures exception of Z2. Significant positive correlation showed by $\mathrm{CV}$ with nearly all measures while of negative with Median. All negative correlations were seen of Median. Measures of set of $\mathrm{S}_{\mathrm{i}}{ }^{1}, \mathrm{~S}_{\mathrm{i}}{ }^{2}, \mathrm{~S}_{\mathrm{i}}{ }^{3}$, $\mathrm{S}_{\mathrm{i}}^{4}, \mathrm{~S}_{\mathrm{i}}{ }^{5}, \mathrm{~S}_{\mathrm{i}}{ }^{6}, \mathrm{~S}_{\mathrm{i}}{ }^{7}$ exhibited positive correlations with other measures as well as among themselves except of negative with CMR, Z1 and $\mathrm{Z} 2$ values. CMR measure maintained negative relationships with most of the measures. CSD, CMR \& CMed had positive relationships with all measures and negative with Z2 only. Seven nonparametric measures based on corrected datasets $\mathrm{CS}_{\mathrm{i}}{ }^{1}, \mathrm{CS}_{\mathrm{i}}{ }^{2}, \mathrm{CS}_{\mathrm{i}}{ }^{3}$, $\mathrm{CS}_{\mathrm{i}}{ }^{4}, \mathrm{CS}_{\mathrm{i}}{ }^{5}, \mathrm{CS}_{\mathrm{i}}{ }^{6}, \mathrm{CS}_{\mathrm{i}}{ }^{7}$ behaved in similar manner as all expressed positive relationships with others and themselves while indirect with measure Z2. The rank of a genotype in a specific environment cannot be judged as per the phenotypic values, because the stability has to be measured independently of the genotypic effect. Therefore, the rank of the ith genotype in the jth environment is estimated based on the corrected phenotypic values (Sabaghnia et al., 2012). Thennarasu (1995) proposed four non-parametric stability measures on the basis of adjusted ranks of genotypes within each test environment. These measures $\mathrm{NP}_{\mathrm{i}}^{(1)}, \mathrm{NP}_{\mathrm{i}}^{(2)}, \mathrm{NP}_{\mathrm{i}}{ }^{(3)}, \mathrm{NP}_{\mathrm{i}}{ }^{(4)}$ had expressed negative relationships with $\mathrm{Z} 1$ and $\mathrm{Z} 2$ otherwise had positive relationships. $\mathrm{Z} 1$ is related to $\mathrm{Z} 2$ in inverse manner.

\section{Biplot graphical analysis}

Biplot analysis of rank-based measures had been carried out to explore if any type of association among measures. Loadings of the first two principal components axes (PCA) of non-parametric measures were shown in table 12 (Mohammadi et al., 2016). Both significant PAC's accounting for $87.7 \%$ of the variations of the original variables. The PCA1 versus PCA2 were used to generate four clusters in the biplot graphical analysis as illustrated in figure 1. Grouping of Yield with GAI as well as of MR and Med were clubbed together in graphical analysis which is otherwise also obvious. Measure CMed \& CMR expressed close affinity with $\mathrm{SD}, \mathrm{NP}_{\mathrm{i}}^{(3)}$, $\mathrm{NP}_{\mathrm{i}}{ }^{(4)} \& \mathrm{~S}_{\mathrm{i}}{ }^{1}, \mathrm{~S}_{\mathrm{i}}{ }^{2}, \mathrm{~S}_{\mathrm{i}}{ }^{3}, \mathrm{~S}_{\mathrm{i}}{ }^{4}, \mathrm{~S}_{\mathrm{i}}{ }^{5}, \mathrm{~S}_{\mathrm{i}}{ }^{6}, \mathrm{~S}_{\mathrm{i}}{ }^{7}$ whereas $\mathrm{CCV}, \mathrm{CSD}, \mathrm{NP}_{\mathrm{i}}{ }^{(1)}$ associated with $\mathrm{CS}_{\mathrm{i}}{ }^{1}, \mathrm{CS}_{\mathrm{i}}{ }^{2}$, $\mathrm{CS}_{\mathrm{i}}{ }^{3}, \mathrm{CS}_{\mathrm{i}}^{4}, \mathrm{CS}_{\mathrm{i}}{ }^{5}, \mathrm{CS}_{\mathrm{i}}{ }^{6}, \mathrm{CS}_{\mathrm{i}}{ }^{7}$ along with $\mathrm{Z} 1$.

\section{Analytic analysis as per BLUE's}

Average yield of wheat genotypes identified G1, G7, G12, whereas GAI selected G1, G12, G9 as genotypes of choice, large values of Median observed for G5, G4, G2 while consistent yield of G8, G12, G4 expressed by least values of standard deviation (Table 5). G4, G5, G3 as genotypes of least variations as pointed by values of coefficient of variation; $\mathrm{S}_{\mathrm{i}}{ }^{1}$ measure selected G8, G12, G4 opposed to $\mathrm{S}_{\mathrm{i}}^{2}$ for G8, G4, G3. Same set of genotypes G4, G8, G12 considered by $\mathrm{S}_{\mathrm{i}}^{3} \& \mathrm{~S}_{\mathrm{i}}{ }^{4}$, while $\mathrm{S}_{\mathrm{i}}^{5}$ favouredG12,G8,G4 whereas $\mathrm{S}_{\mathrm{i}}{ }^{6}$ measure identified $\mathrm{G} 4, \mathrm{G} 5, \mathrm{G} 3 \& \mathrm{~S}_{\mathrm{i}}^{7}$ pointed towards G8, G12, G4 wheat genotypes. Average median of ranks as per corrected yield values selected G7, G10, G4 and corrected standard deviation observed suitability of G12, G8, G4 genotypes. Coefficient of variation as per corrected yield values exhibited G8, G12, G4 while median values for G5, G6, G9 and G12, G8, G11 by $\mathrm{CS}_{\mathrm{i}}{ }^{1}, \mathrm{G} 12, \mathrm{G} 8, \mathrm{G} 9$ by $\mathrm{CS}_{\mathrm{i}}{ }^{2}, \mathrm{CS}_{\mathrm{i}}{ }^{3}$ for $\mathrm{G} 8, \mathrm{G} 12, \mathrm{G} 4$ \& as per $\mathrm{CS}_{\mathrm{i}}{ }^{4}$ values G12,G8,G4 while G12,G8, G11 by $\mathrm{CS}_{\mathrm{i}}{ }^{5}$ \& G8,G12, G4 by $\mathrm{CS}_{\mathrm{i}}^{6}$ values and lastly $\mathrm{CS}_{\mathrm{i}}{ }^{7}$ pointed towards G12, G8, G4 (table 6). No parametric measures $\mathrm{NP}_{\mathrm{i}}{ }^{(1)}$ for $(\mathrm{G} 12, \mathrm{G} 8, \mathrm{G} 11)$; $\mathrm{NP}_{\mathrm{i}}^{(2)}, \mathrm{NP}_{\mathrm{i}}^{(3)}, \mathrm{NP}_{\mathrm{i}}{ }^{(4)}$ considered ranks of genotypes by original and corrected values 
also selected G4, G5, G8 wheat genotypes and G1 along with G6 would be of unsuitable type as per value of $\mathrm{NP}_{\mathrm{i}}^{(1)}, \mathrm{NP}_{\mathrm{i}}^{(2)}, \mathrm{NP}_{\mathrm{i}}^{(3)}, \mathrm{NP}_{\mathrm{i}}$ (4) whereas values of measures $\mathrm{Z} 1$ observed G9, G3, G4 and genotypes G3, G9, G11 as per $\mathrm{Z} 2$ values.

Calculated value of $\chi^{2}=97.6$ is less than table of $\chi^{2}(0.05,290)=124.3(135.8)$, resulted an overall similarity among non parametric measures. Values of both measures : $\mathrm{Z}_{1}$ sum $=$ 17.89 and $Z_{2}$ sum $=16.8$ (Table 6) were less than the critical value of $\chi^{2}(0.05,29)=42.6$. This indicated the non-significant differences among genotypes as per ranks of $\mathrm{CS}_{\mathrm{i}}{ }^{1}$ and $\mathrm{CS}_{\mathrm{i}}^{2}$ measures. More over the individual $\mathrm{Z}$ values showed HP1963, \&WH1202were significantly unstable relative to others, with their individual $\mathrm{Z}$ values more than the critical value of $\chi^{2}(0.05,1)=3.84$.

\section{Association analysis}

As per values of rank correlation among nonparametric measures, highly significant $\left(\mathrm{p}^{<}\right.$ 0.01) positive correlation of yield observed with GAI, Med and negative correlation with $\mathrm{MR}, \mathrm{CV}, \mathrm{S}_{\mathrm{i}}{ }^{3}, \mathrm{~S}_{\mathrm{i}}{ }^{6}, \mathrm{NP}_{\mathrm{i}}{ }^{(2)}, \mathrm{NP}_{\mathrm{i}}{ }^{(3)}, \mathrm{NP}_{\mathrm{i}}{ }^{(4)}$ (Table 13). Measure GAI showed negative correlations with most of the measures and positive with Med only. Mean rank (MR) expressed positive correlation with $\mathrm{CV}, \mathrm{S}_{\mathrm{i}}{ }^{3}$, $\mathrm{S}_{\mathrm{i}}{ }^{6}, \mathrm{NP}_{\mathrm{i}}{ }^{(2)}, \mathrm{NP}_{\mathrm{i}}{ }^{(3)}, \mathrm{NP}_{\mathrm{i}}{ }^{(4)}$ and negative with Med. Measure SD maintained highly significant and significant direct relations with almost all the measures exception of $S_{i}{ }^{3} \& S_{i}{ }^{6}$ i.e. perfect positive correlation. $\mathrm{CV}$ showed significant positive correlation with $\mathrm{S}_{\mathrm{i}}{ }^{3}, \mathrm{~S}_{\mathrm{i}}{ }^{6}, \mathrm{NP}_{\mathrm{i}}{ }^{(2)}, \mathrm{NP}_{\mathrm{i}}{ }^{(3)}$, $\mathrm{NP}_{\mathrm{i}}{ }^{(4)}$ and negative with Median. All negative correlations of significance and others were seen of Median. Set of $\mathrm{S}_{\mathrm{i}}{ }^{1}, \mathrm{~S}_{\mathrm{i}}{ }^{2}, \mathrm{~S}_{\mathrm{i}}{ }^{3}, \mathrm{~S}_{\mathrm{i}}{ }^{4}, \mathrm{~S}_{\mathrm{i}}{ }^{5}, \mathrm{~S}_{\mathrm{i}}{ }^{6}$, $\mathrm{S}_{\mathrm{i}}{ }^{7}$ behaved in similar manner and exhibited positive correlation with other measures as well as among themselves except of negative with $\mathrm{CMR}, \mathrm{Z} 1$ and $\mathrm{Z} 2$ values. CMR measure maintained weak negative relationships with all the measures. CSD, CMR \&CMed had expressed positive correlations with measures and only significant negative with $\mathrm{Z} 2$ values. Seven non parametric measures based on corrected yield values of genotypes i.e. $\mathrm{CS}_{\mathrm{i}}{ }^{1}, \mathrm{CS}_{\mathrm{i}}{ }^{2}, \mathrm{CS}_{\mathrm{i}}{ }^{3}, \mathrm{CS}_{\mathrm{i}}{ }^{4}, \mathrm{CS}_{\mathrm{i}}{ }^{5}, \mathrm{CS}_{\mathrm{i}}{ }^{6}$ and $\mathrm{CS}_{\mathrm{i}}{ }^{7}$ expressed positive relationships with others and themselves while highly significant indirect with $\mathrm{Z} 2$ values. Measures $\mathrm{NP}_{\mathrm{i}}{ }^{(1)}$, $\mathrm{NP}_{\mathrm{i}}^{(2)}, \mathrm{NP}_{\mathrm{i}}^{\left({ }^{(3)}\right.}, \mathrm{NP}_{\mathrm{i}}^{(4)}$ had expressed negative relationships with $\mathrm{Z} 2$ otherwise maintained positive relationships. An inverse relationship has evident between $\mathrm{Z} 1 \& \mathrm{Z} 2$ values.

\section{Biplot graphical analysis}

Biplot analysis based on first two principal components had expressed five clusters of 30 non parametric measures. Loadings of the first two principal components axes (PCA) of ranks of non-parametric measures were shown in table 14. Both significant PAC's accounting for $85.3 \%$ of the variance of the original variables (Figure 2). Grouping of Yield with GAI along with Z1 \& Z2 whereas MR with Med are observed in graphical analysis. CV expressed affinity with CMR, $\mathrm{S}_{\mathrm{i}}{ }^{3}, \mathrm{~S}_{\mathrm{i}}{ }^{6}, \mathrm{NP}_{\mathrm{i}}{ }^{(2)}, \mathrm{NP}_{\mathrm{i}}{ }^{(3)} \& \mathrm{NP}_{\mathrm{i}}^{(4)}$ whereas SD grouped with $\mathrm{S}_{\mathrm{i}}{ }^{1}, \mathrm{~S}_{\mathrm{i}}{ }^{2}, \mathrm{~S}_{\mathrm{i}}^{4}, \mathrm{~S}_{\mathrm{i}}{ }^{5}, \mathrm{~S}_{\mathrm{i}}^{7}$ measures. Separate cluster of $\mathrm{NP}_{i}^{(1)}, \mathrm{CSD}, \mathrm{CCV}$, $\mathrm{CS}_{\mathrm{i}}{ }^{1}, \mathrm{CS}_{\mathrm{i}}{ }^{2}, \mathrm{CS}_{\mathrm{i}}{ }^{3}, \mathrm{CS}_{\mathrm{i}}{ }^{4}, \mathrm{CS}_{\mathrm{i}}{ }^{5}, \mathrm{CS}_{\mathrm{i}}{ }^{6}$ andCS ${ }_{\mathrm{i}}{ }^{7}$.

\section{Second year of study 2017-18}

\section{Analytic analysis as per BLUP's}

Mean wheat yield of genotypes observed, G4 as the highest yielding with $58 \mathrm{q} /$ ha followed by $\mathrm{G} 2$ and $\mathrm{G} 7$, though remarkable yield differences were observed among the genotypes (Table 7). Measure GAI selected G4, G2, G7 as genotypes with higher adaptable index values. MR pointed towards G5, G14, G11 and SD for G9,G14, G15 whereas CV for G5,G14, G15 as genotypes of stable performance, while G5, G15 based on 
MR \& GAI, G7, G4 based on SD and CV, would be unstable genotypes. These descriptive statistics based on ranks can be used for genotype comparative evaluation. Values of Median selected G4, G2, G7 genotypes.

Seven nonparametric measures based on original grain yield of genotypes $\left(\mathrm{S}_{\mathrm{i}}{ }^{1}, \mathrm{~S}_{\mathrm{i}}{ }^{2}, \mathrm{~S}_{\mathrm{i}}{ }^{3}, \mathrm{~S}_{\mathrm{i}}^{4}, \mathrm{~S}_{\mathrm{i}}^{5}, \mathrm{~S}_{\mathrm{i}}{ }^{6}\right.$ and $\left.\mathrm{S}_{\mathrm{i}}{ }^{7}\right)$ indicated that (G9, G15, G14), (G9, G8, G6), (G9,G13,G15), (G9, G13, G1), (G9, G13, G1), (G9, G15,G13), (G9, G13, G1) as sets of genotypes respectively. According to corrected yield (table 8), G1,G8 \&G10 by mean of corrected ranks (CMR), G12,G11 $\& \mathrm{G} 8$ by standard deviation of corrected ranks (CSD) and G8,G10 \&G4were the stable as per coefficient of variation of corrected ranks (CCV). Nonparametric measures of stability based on corrected yield values $\left(\mathrm{CS}_{\mathrm{i}}{ }^{1}, \mathrm{CS}_{\mathrm{i}}{ }^{2}\right.$, $\left.\mathrm{CS}_{\mathrm{i}}{ }^{3}, \mathrm{CS}_{\mathrm{i}}{ }^{4}, \mathrm{CS}_{\mathrm{i}}{ }^{5}, \mathrm{CS}_{\mathrm{i}}{ }^{6}, \mathrm{CS}_{\mathrm{i}}{ }^{7}\right)$ identified stable genotypes (G14, G13, G9), (G14, G11, G9), (G14, G8, G1), (G14, G13, G8), (G8, G13, G14), (G14, G8, G1), (G14, G13, G8) and G7,G10 were of unstable type as per these nonparametric measures. First measure $\mathrm{NP}_{\mathrm{i}}{ }^{(1)}$ considered genotypes G9, G14 and G13 were of stable yield. Genotypes G14, G11 and G15 had expressed the lower values of $\mathrm{NP}_{\mathrm{i}}^{(2)}$, $\mathrm{NP}_{\mathrm{i}}{ }^{(3)} \& \mathrm{NP}_{\mathrm{i}}{ }^{(4)}$, whereas $\mathrm{Z1}$ and $\mathrm{Z} 2$ pointed for G15,G11, G6 as suitable as well as G7 \&G10 would be of unsuitable performance.

Value of the the $\chi^{2}$ statistics was less than table value of $\chi^{2}(0.01,290)=135.8$, which resulted an overall similarity among nonparametric measures. Values of both measures $Z_{1}$ sum $=17.29$ and $Z_{2}$ sum $=18.66$ (Table 8) were less than the critical value of $\chi^{2}(0.05,29)=42.6$. This indicated the nonsignificant differences among genotypes as per ranks of $\mathrm{CS}_{\mathrm{i}}{ }^{1}$ and $\mathrm{CS}_{\mathrm{i}}{ }^{2}$ measures. The individual $\mathrm{Z}$ values showed DBW 233was significantly unstable as compared to studied genotypes as $\mathrm{Z}$ values more than the critical value of $\chi^{2}(0.05,1)=3.84$.

\section{Association analysis}

Spearman's rank correlation values among non-parametric measures had reflected in table 15 as based on BLUP's of genotypes yield across environments. Yield had highly significant positive correlation with GAI, Med and negative correlation with MR,SD, $\mathrm{CV}, \mathrm{S}_{\mathrm{i}}{ }^{3}, \mathrm{~S}_{\mathrm{i}}{ }^{6}, \mathrm{NP}_{\mathrm{i}}{ }^{(2)}, \mathrm{NP}_{\mathrm{i}}{ }^{(3)}, \mathrm{NP}_{\mathrm{i}}{ }^{(4)}$ along with weak direct relationships with CMR, Z2 \& negative with other measures $\mathrm{SD}, \mathrm{S}_{\mathrm{i}}{ }^{2}, \mathrm{~S}_{\mathrm{i}}{ }^{4}, \mathrm{~S}_{\mathrm{i}}{ }^{5}$, $\mathrm{S}_{\mathrm{i}}{ }^{7}, \mathrm{CSD}, \mathrm{CCV}, \mathrm{CS}_{\mathrm{i}}{ }^{1}, \mathrm{CS}_{\mathrm{i}}{ }^{2}, \mathrm{CS}_{\mathrm{i}}{ }^{3}, \mathrm{CS}_{\mathrm{i}}{ }^{4}, \mathrm{CS}_{\mathrm{i}}{ }^{5}, \mathrm{CS}_{\mathrm{i}}{ }^{6}$ and $\mathrm{CS}_{\mathrm{i}}{ }^{7}$. Measure GAI mentioned negative correlations with MR, $\mathrm{SD}, \mathrm{CV}, \mathrm{S}_{\mathrm{i}}{ }^{3}, \mathrm{~S}_{\mathrm{i}}{ }^{6}, \mathrm{NP}_{\mathrm{i}}{ }^{(2)}$, $\mathrm{NP}_{\mathrm{i}}^{(3)}, \mathrm{NP}_{\mathrm{i}}{ }^{(4)}$ and positive with median. Mean rank (MR) expressed positive correlations with all measures though degree of correlations vary except negative with Median. SD also expressed positive correlation with measures with Median and Z2 as exceptions. Similarly, CV exhibited same type of behavior. Median expressed only negative correlations with variable degree of association. Measures CMR and Z2 showed negative correlations with $\mathrm{S}_{\mathrm{i}}{ }^{1}, \mathrm{~S}_{\mathrm{i}}{ }^{2}, \mathrm{~S}_{\mathrm{i}}{ }^{3}$, $\mathrm{Si}^{4}, \mathrm{Si}^{5}, \mathrm{~S}_{\mathrm{i}}^{7}$ otherwise only direct relationships were noticed. CMR showed only significant negative correlation with CMed. Measures CSD, CMR \&CMed had significant positive relationships with all measures and negative with Z2 only. Set of $\mathrm{CS}_{\mathrm{i}}{ }^{1}, \mathrm{CS}_{\mathrm{i}}{ }^{2}, \mathrm{CS}_{\mathrm{i}}{ }^{3}, \mathrm{CS}_{\mathrm{i}}{ }^{4}$, $\mathrm{CS}_{\mathrm{i}}{ }^{5}, \mathrm{CS}_{\mathrm{i}}{ }^{6}$ and $\mathrm{CS}_{\mathrm{i}}{ }^{7}$ behaved in similar manner as all expressed positive relationships with others and themselves while indirect with Z2.Non-parametric measures considered adjusted ranks of genotypes within each test environment. These measures $\mathrm{NP}_{\mathrm{i}}^{(1)}, \mathrm{NP}_{\mathrm{i}}^{(2)}$, $\mathrm{NP}_{\mathrm{i}}{ }^{(3)}, \quad \mathrm{NP}_{\mathrm{i}}{ }^{(4)}$ had expressed negative relationships with $\mathrm{Z} 1$ and $\mathrm{Z} 2$ otherwise had positive relationships. $\mathrm{Z} 1$ is related to $\mathrm{Z} 2$ in weakly but inverse manner. 
Table.1 Parentage details of genotypes and environmental conditions (2016-17)

\begin{tabular}{|c|c|c|c|c|c|c|c|}
\hline Code & Genotype & Parentage & Code & Environments & Latitude & Longitude & $\begin{array}{l}\text { Altitude } \\
\text { (m) }\end{array}$ \\
\hline G1 & DBW189 & $\begin{array}{l}\text { (KACHU\#1/4/CROC_1/AE.SQUARROSA(205)//BORL95/3/2*MILAN } \\
\text { /5/KACHU) }\end{array}$ & E1 & Alwar & $27^{\circ} 7^{\prime} \mathrm{N}$ & $76^{\circ} 1^{\prime} \mathrm{E}$ & 271 \\
\hline G2 & DBW196 & $(\mathrm{ROLF} 07 * 2 / \mathrm{KACHU} \# 1)$ & $\mathrm{E} 2$ & Bareilly & $28^{\circ} 22^{\prime} \mathrm{N}$ & $79^{\circ} 24^{\prime} \mathrm{E}$ & 172.9 \\
\hline G3 & PBW750 & $\begin{array}{l}\text { (TOB/ERA/TOB/CNO67/3/PLO/4/VEE\#5/5/KAUZ/6/FRET2/7/PASTOR// } \\
\text { MILAN/KAUZ/3/BAV92) }\end{array}$ & E3 & Bawal & $28^{\circ} 10^{\prime} \mathrm{N}$ & $76^{\circ} 50^{\prime} \mathrm{E}$ & 266 \\
\hline G4 & WH1202 & (D67.2/PARANA66.270//AE.SQ.(320)/3/CUNNINGHAM) & E4 & Bathinda & $30^{\circ} 09^{\prime} \mathrm{N}$ & $74^{\circ} 55^{\prime} \mathrm{E}$ & 211 \\
\hline G5 & HD3226 & (GRACKLE/HD2894) & E5 & Bikaner & $28^{\circ} 02^{\prime} \mathrm{N}$ & $73^{\circ} 30^{\prime} \mathrm{E}$ & 230 \\
\hline G6 & UP2942 & $\begin{array}{l}\text { (CS/TH.SC//3*PVN/3/MIRLO/BUC/4/URES/JUN//KAUZ/5/HUITES/6/ } \\
\text { YANAC/7/CS/ TH.SC//3*PVN/3/MIRLO/BUC/4/MILAN/5/TILHI) }\end{array}$ & E6 & Bulandshahr & $28^{\circ} 24^{\prime} \mathrm{N}$ & $77^{\circ} 50 ’ \mathrm{E}$ & 204 \\
\hline G7 & HP1963 & $\begin{array}{l}\text { (FRET2/TUKURU//FRET2/3/MUNIA/CHTO//AMSEL/4/FRET2/TUKURU//FRET } \\
\text { 2) }\end{array}$ & E7 & Delhi & $28^{\circ} 38^{\prime} \mathrm{N}$ & $77^{\circ} 13^{\prime} \mathrm{E}$ & 222 \\
\hline G8 & BRW3773 & (FRANCOLIN\#1//WBLL1*2/BRAMBLING/3/WBLL1*2) & E8 & Dhakrani & $30.44 \mathrm{~N}$ & 77.74 'E & 452 \\
\hline G9 & HD2967 & (ALD/CUC//URES/HD2160M/HD2278) & E9 & Dhaulakuan & $30^{\circ} 16^{\prime} \mathrm{N}$ & $74^{\circ} 56^{\prime} \mathrm{E}$ & 236 \\
\hline G10 & WH1105 & (MILAN/S87230//BABAX ) & E10 & Durgapura & $26^{\circ} 51^{\prime} \mathrm{N}$ & $75^{\circ} 47^{\prime} \mathrm{E}$ & 390 \\
\hline G11 & DBW88 & (KAUZ//ALTAR84/AOS/3/MILAN/KAUZ/4/HUITES) & E11 & Faridkot & $30^{\circ} 40^{\prime} \mathrm{N}$ & $74^{\circ} 04^{\prime} \mathrm{E}$ & 200 \\
\hline \multirow[t]{17}{*}{ G12 } & HD 3086 & (DBW14/HD2733//HUW468) & E12 & Gurdaspur & $32^{\circ} 2 \mathrm{~N}$ & $75^{\circ} 24^{\prime} \mathrm{E}$ & 265 \\
\hline & & & E13 & Hanumangarh & $29^{\circ} 62^{\prime} \mathrm{N}$ & $74^{\circ} 28^{\prime} \mathrm{E}$ & 177 \\
\hline & & & E14 & Hisar & $29^{\circ} 19^{\prime} \mathrm{N}$ & $75^{\circ} 43^{\prime} \mathrm{E}$ & 215 \\
\hline & & & E15 & Jammu & $32^{\circ} 43^{\prime} \mathrm{N}$ & $74^{\circ} 51^{\prime} \mathrm{E}$ & 351 \\
\hline & & & E16 & Jodhpur & $26^{\circ} 26^{\prime} \mathrm{N}$ & $73^{\circ} 00^{\prime} \mathrm{E}$ & 235 \\
\hline & & & E17 & Karnal & $29^{\circ} 43^{\prime} \mathrm{N}$ & $70^{\circ} 58^{\prime} \mathrm{E}$ & 245 \\
\hline & & & E18 & Kashipur & $29^{\circ} 21^{\prime} \mathrm{N}$ & $78^{\circ} 96^{\prime} \mathrm{E}$ & 218 \\
\hline & & & E19 & Kapurthala & $31^{\circ} 22^{\prime} \mathrm{N}$ & $75^{\circ} 22^{\prime} \mathrm{E}$ & 229 \\
\hline & & & E20 & Ludhiana & $30^{\circ} 54^{\prime} \mathrm{N}$ & $75^{\circ} 51^{\prime} \mathrm{E}$ & 252 \\
\hline & & & E21 & Modipuram & $29^{\circ} 43^{\prime} \mathrm{N}$ & $77^{\circ} 42^{\prime} \mathrm{E}$ & 237 \\
\hline & & & E22 & Nagina & $29^{\circ} 28^{\prime} \mathrm{N}$ & $78^{\circ} 32^{\prime} \mathrm{E}$ & 245 \\
\hline & & & E23 & Pantnagar & $29^{\circ} 05^{\prime} \mathrm{N}$ & $79^{\circ} 29^{\prime} \mathrm{E}$ & 236.54 \\
\hline & & & E24 & Rauni & $30^{\circ} 90^{\prime} \mathrm{N}$ & $75^{\circ} 85^{\prime} \mathrm{E}$ & 262 \\
\hline & & & E25 & Rohtak & $28^{\circ} 53^{\prime} \mathrm{N}$ & $76^{\circ} 35^{\prime} \mathrm{E}$ & 222.5 \\
\hline & & & E26 & Rampur-KVK & $28^{\circ} 48^{\prime} \mathrm{N}$ & $79^{\circ} 10^{\prime} \mathrm{E}$ & 1021 \\
\hline & & & E27 & Sriganganagar & $29^{\circ} 66^{\prime} \mathrm{N}$ & $75^{\circ} 53^{\prime} \mathrm{E}$ & 175.6 \\
\hline & & & E28 & Shikopur & $29^{\circ} 10^{\prime} \mathrm{N}$ & $77^{\circ} 27^{\prime} \mathrm{E}$ & 217 \\
\hline
\end{tabular}


Table.2 Parentage details of wheat genotypes along with environmental conditions (2017-18)

\begin{tabular}{|c|c|c|c|c|c|c|c|}
\hline Code & Genotype & Parentage & Code & Environments & Latitude & Longitude & $\begin{array}{l}\text { Altitude } \\
\text { (m) }\end{array}$ \\
\hline G1 & UP2981 & (CHYAK/PAURAQ) & E1 & Delhi & $28^{\circ} 4^{\prime} \mathrm{N}$ & $77^{\circ} 13^{\prime} \mathrm{E}$ & 228 \\
\hline G2 & DBW221 & (36IBWSN284/22ESWYT28) & $\mathrm{E} 2$ & Faridkot & $30^{\circ} 40^{\prime} \mathrm{N}$ & $74^{\circ} 04^{\prime} \mathrm{E}$ & 200 \\
\hline G3 & DBW222 & $\begin{array}{l}\text { (KACHU/SAUAL/8/ATTILA*2/PBW65/6/PVN//CAR422/ANA/5/BOW/CRO } \\
\text { W//BUC/PVN/3/YR/4/TRAP\#1/7/ATTILA/2*PASTOR) }\end{array}$ & E3 & Gurdaspur & $30^{\circ} 02^{\prime} \mathrm{N}$ & $75^{\circ} 24^{\prime} \mathrm{E}$ & 265 \\
\hline G4 & BRW3792 & (PF74354//LD/ALD/4/2*BR12*2/3/JUP//PAR214) & $\mathrm{E} 4$ & Bathinda & $30^{\circ} 09^{\prime} \mathrm{N}$ & $74^{\circ} 55^{\prime} \mathrm{E}$ & 211 \\
\hline G5 & PBW763 & $\begin{array}{l}\text { (PBW621/3/YR10/6*AVOCET//4*PBW343/4/2*PBW621/5/PBW621/3/YR15/ } \\
6 * \text { AVOCET//4*PBW343/4/2*PBW621) }\end{array}$ & E5 & Kapurthala & $31^{\circ} 22^{\prime} \mathrm{N}$ & $75^{\circ} 22^{\prime} \mathrm{E}$ & 229 \\
\hline G6 & PBW766 & (NAC/TH.AC//3*PVN/3/MIRLO/BUC/4/2*PASTOR/5/KACHU/6/KACHU) & E6 & Ludhiana & $30^{\circ} 54^{\prime} \mathrm{N}$ & $75^{\circ} 48^{\prime} \mathrm{E}$ & 247 \\
\hline G7 & DBW233 & (CHIBIA//PRLII/CM65531/3/SKAUZ/BAV92/4/MUNAL\#1) & E7 & Rauni & $30^{\circ} 90^{\prime} \mathrm{N}$ & $75^{\circ} 85^{\prime} \mathrm{E}$ & 262 \\
\hline G8 & HD3226 & (GRACKLE/HD2894) & E8 & Hisar & $29^{\circ} 10^{\prime} \mathrm{N}$ & $75^{\circ} 46^{\prime} \mathrm{E}$ & 229 \\
\hline G9 & PBW801 & $\begin{array}{l}\text { (PBW621/3/Yr10/6*Avocet//4*PBW 343/4/2*PBW 621/5/PBW 621/3/Yr } \\
\text { 15/6*Avocet// 4*PBW 343/4/2*PBW 621) }\end{array}$ & E9 & Bawal & $28^{\circ} 10^{\prime} \mathrm{N}$ & $76^{\circ} 50^{\prime} \mathrm{E}$ & 266 \\
\hline G10 & PBW800 & $\begin{array}{l}\text { (HD 2967/4/BW 9250*3// Yr10/6*Avocet/3/ BW } \\
\text { 9250*3//Yr15/6*Avocet/5/2*HD 2967) }\end{array}$ & E10 & Karnal & $29^{\circ} 43^{\prime} \mathrm{N}$ & $70^{\circ} 58^{\prime} \mathrm{E}$ & 245 \\
\hline G11 & DPW621-50 & (KAUZ//ALTAR84/AOS/3/MILAN/KAUZ/4/HUITES) & E11 & Rohtak & $28^{\circ} 53^{\prime} \mathrm{N}$ & $76^{\circ} 35^{\prime} \mathrm{E}$ & 222.5 \\
\hline G12 & HD3086 & (DBW14/HD2733//HUW468) & E12 & Jammu & $32^{\circ} 40^{\prime} \mathrm{N}$ & $74^{\circ} 54^{\prime} \mathrm{E}$ & 356 \\
\hline G13 & HD 2967 & (ALD/CUC//URES/HD2160M/HD2278) & E13 & Pantnagar & $29^{\circ} 02^{\prime} \mathrm{N}$ & $79^{\circ} 48^{\prime} \mathrm{E}$ & 243.8 \\
\hline G14 & DBW 88 & (KAUZ//ALTAR84/AOS/3/MILAN/KAUZ/4/HUITES) & E14 & Kashipur & $29^{\circ} 21^{\prime} \mathrm{N}$ & $78^{\circ} 96^{\prime} \mathrm{E}$ & 218 \\
\hline \multirow[t]{11}{*}{ G15 } & WH 1105 & (MILAN/S87230//BABAX ) & E15 & Bulandshahr & $28^{\circ} 40^{\prime} \mathrm{N}$ & $77^{\circ} 84^{\prime} \mathrm{E}$ & 195 \\
\hline & & & E16 & Bareilly & $28^{\circ} 22^{\prime} \mathrm{N}$ & $79^{\circ} 24^{\prime} \mathrm{E}$ & 172.9 \\
\hline & & & E17 & Nagina & $29^{\circ} 28^{\prime} \mathrm{N}$ & $78^{\circ} 32^{\prime} \mathrm{E}$ & 245 \\
\hline & & & E18 & Sriganganagar & $29^{\circ} 66^{\prime} \mathrm{N}$ & $75^{\circ} 53^{\prime} \mathrm{E}$ & 175.6 \\
\hline & & & E19 & Tabiji & $26^{\circ} 35^{\prime} \mathrm{N}$ & $74^{\circ} 61^{\prime} \mathrm{E}$ & 508 \\
\hline & & & E20 & Kotputli & $27^{\circ} 42^{\prime} \mathrm{N}$ & $76^{\circ} 12^{\prime} \mathrm{E}$ & 362 \\
\hline & & & E21 & Hanumangarh & $29^{\circ} 62^{\prime} \mathrm{N}$ & $74^{\circ} 28^{\prime} \mathrm{E}$ & 177 \\
\hline & & & E22 & Jodhpur & $26^{\circ} 26^{\prime} \mathrm{N}$ & $73^{\circ} 00^{\circ} \mathrm{E}$ & 235 \\
\hline & & & E23 & Alwar & $27^{\circ} 7^{\prime} \mathrm{N}$ & $76^{\circ} 1^{\prime} \mathrm{E}$ & 271 \\
\hline & & & E24 & Bikaner & $28^{\circ} 02^{\prime} \mathrm{N}$ & $73^{\circ} 30^{\prime} \mathrm{E}$ & 230 \\
\hline & & & E25 & Durgapura & $26^{\circ} 51{ }^{\prime} \mathrm{N}$ & $75^{\circ} 47^{\prime} \mathrm{E}$ & 390 \\
\hline
\end{tabular}


Table.3 Non parametric measures of genotypes based on original values of yield 2016-17 (BLUP)

\begin{tabular}{|c|c|c|c|c|c|c|c|c|c|c|c|c|c|c|c|}
\hline blup16-17 & Yield & GAI & MR & SD & CV & Med & $\mathbf{S}_{\mathrm{i}}{ }^{1}$ & $\mathbf{S}_{\mathrm{i}}^{2}$ & $\mathbf{S}_{\mathrm{i}}{ }^{3}$ & $S_{i}^{4}$ & $S_{i}^{5}$ & $S_{i}{ }^{6}$ & $\mathbf{S}_{\mathrm{i}}{ }^{7}$ & CMR & CSD \\
\hline BRW3773 & 57.43 & 56.82 & 5.57 & 3.99 & 0.717 & 5 & 4.56 & 15.96 & 77.33 & 3.92 & 3.41 & 17.13 & 4.51 & 7.25 & 4.29 \\
\hline DBW189 & 55.25 & 54.57 & 7.50 & 3.20 & 0.427 & 8.5 & 3.62 & 10.26 & 36.93 & 3.15 & 2.71 & 10.13 & 3.64 & 6.68 & 3.46 \\
\hline DBW196 & 54.78 & 54.06 & 7.50 & 3.01 & 0.402 & 8 & 3.47 & 9.07 & 32.67 & 2.96 & 2.54 & 9.47 & 3.45 & 6.07 & 3.28 \\
\hline DBW88 & 55.04 & 54.52 & 8.21 & 2.99 & 0.363 & 9 & 3.33 & 8.92 & 29.30 & 2.93 & 2.54 & 8.66 & 3.38 & 6.86 & 3.14 \\
\hline HD2967 & 51.86 & 51.07 & 9.32 & 3.66 & 0.393 & 11.5 & 3.78 & 13.41 & 38.85 & 3.60 & 3.09 & 9.27 & 4.19 & 6.21 & 4.65 \\
\hline HD3086 & 57.16 & 56.33 & 4.71 & 3.56 & 0.755 & 4 & 4.03 & 12.66 & 72.48 & 3.49 & 3.03 & 18.00 & 4.03 & 6.46 & 3.67 \\
\hline HD3226 & 57.23 & 56.39 & 4.96 & 3.17 & 0.638 & 4 & 3.57 & 10.04 & 54.58 & 3.11 & 2.74 & 15.47 & 3.53 & 6.68 & 3.53 \\
\hline HP1963 & 56.85 & 56.23 & 5.71 & 2.71 & 0.474 & 6 & 3.07 & 7.32 & 34.60 & 2.66 & 2.09 & 10.25 & 3.38 & 6.96 & 2.97 \\
\hline PBW750 & 56.98 & 56.24 & 5.00 & 3.19 & 0.637 & 4 & 3.57 & 10.15 & 54.80 & 3.13 & 2.57 & 14.40 & 3.81 & 6.29 & 3.41 \\
\hline UP2942 & 55.80 & 55.09 & 7.39 & 2.71 & 0.367 & 8 & 3.01 & 7.36 & 26.87 & 2.66 & 2.01 & 7.60 & 3.53 & 6.93 & 3.03 \\
\hline WH1105 & 55.14 & 54.41 & 7.29 & 2.54 & 0.348 & 8 & 2.84 & 6.43 & 23.84 & 2.49 & 1.96 & 7.53 & 3.17 & 5.54 & 2.97 \\
\hline WH1202 & 56.99 & 56.39 & 4.82 & 3.06 & 0.634 & 4.5 & 3.46 & 9.34 & 52.29 & 3.00 & 2.46 & 14.31 & 3.65 & 6.07 & 2.94 \\
\hline
\end{tabular}

Table.4 Non parametric measures of genotypes based on corrected values of yield (BLUP)

\begin{tabular}{|c|c|c|c|c|c|c|c|c|c|c|c|c|c|c|c|}
\hline blup16-17 & $\mathrm{CCV}$ & CMed & $\mathrm{CS}_{\mathrm{i}}{ }^{1}$ & $\mathrm{CS}_{\mathrm{i}}{ }^{2}$ & $\mathrm{CS}_{\mathrm{i}}{ }^{3}$ & $\mathrm{CS}_{\mathrm{i}}{ }^{4}$ & $\mathrm{CS}_{\mathrm{i}}{ }^{5}$ & $\mathrm{CS}_{\mathrm{i}}{ }^{6}$ & $\mathrm{CS}_{\mathrm{i}}{ }^{7}$ & $\mathbf{N P}_{\mathbf{i}}^{(1)}$ & $\mathbf{N P}_{\mathbf{i}}^{(2)}$ & $\mathbf{N P}_{\mathbf{i}}{ }^{(3)}$ & $\mathbf{N P}_{\mathbf{i}}^{(4)}$ & Z1 & $\mathbf{Z 2}$ \\
\hline BRW3773 & 0.592 & 8.5 & 4.90 & 18.42 & 68.59 & 4.21 & 3.88 & 12.76 & 4.58 & 3.82 & 0.764 & 0.756 & 0.880 & 6.78 & 9.72 \\
\hline DBW189 & 0.519 & 7 & 4.01 & 12.00 & 48.53 & 3.40 & 3.01 & 12.04 & 3.85 & 2.96 & 0.349 & 0.454 & 0.534 & 0.01 & 0.00 \\
\hline DBW196 & 0.540 & 6.5 & 3.80 & 10.74 & 47.74 & 3.22 & 2.79 & 12.00 & 3.72 & 2.79 & 0.348 & 0.429 & 0.507 & 0.22 & 0.32 \\
\hline DBW88 & 0.457 & 7 & 3.65 & 9.83 & 38.71 & 3.08 & 2.65 & 10.61 & 3.57 & 2.64 & 0.294 & 0.375 & 0.444 & 0.84 & 1.00 \\
\hline HD2967 & 0.748 & 6.5 & 5.22 & 21.58 & 93.77 & 4.56 & 4.36 & 18.77 & 4.78 & 4.36 & 0.379 & 0.489 & 0.560 & 12.25 & 21.49 \\
\hline HD3086 & 0.567 & 6 & 4.28 & 13.44 & 56.15 & 3.60 & 3.07 & 14.32 & 4.22 & 3.04 & 0.759 & 0.764 & 0.907 & 0.73 & 0.54 \\
\hline HD3226 & 0.528 & 7.5 & 4.07 & 12.45 & 50.33 & 3.46 & 3.20 & 11.95 & 3.75 & 3.18 & 0.795 & 0.698 & 0.820 & 0.08 & 0.07 \\
\hline HP1963 & 0.427 & 7.5 & 3.45 & 8.85 & 34.31 & 2.92 & 2.47 & 9.22 & 3.46 & 2.46 & 0.411 & 0.511 & 0.603 & 2.16 & 2.16 \\
\hline PBW750 & 0.542 & 5.5 & 3.95 & 11.62 & 49.91 & 3.35 & 2.90 & 14.75 & 3.87 & 2.86 & 0.714 & 0.669 & 0.789 & 0.00 & 0.02 \\
\hline UP2942 & 0.437 & 7 & 3.48 & 9.18 & 35.77 & 2.98 & 2.37 & 9.49 & 3.73 & 2.36 & 0.295 & 0.402 & 0.471 & 1.89 & 1.72 \\
\hline WH1105 & 0.537 & 5.5 & 3.37 & 8.85 & 43.17 & 2.92 & 2.32 & 11.82 & 3.68 & 2.32 & 0.290 & 0.401 & 0.463 & 2.81 & 2.16 \\
\hline \multirow[t]{3}{*}{ WH1202 } & 0.485 & 6 & 3.40 & 8.66 & 38.52 & 2.89 & 2.37 & 11.07 & 3.52 & 2.36 & 0.524 & 0.599 & 0.705 & 2.60 & 2.44 \\
\hline & & $\mathrm{E}\left(\mathrm{S}^{1}\right)$ & 3.97 & $\mathrm{~V}\left(\mathrm{~S}^{1}\right)$ & 0.1276 & $\mathrm{~W}=$ & 0.338 & $\chi^{2}=$ & 111.594 & & & & & $\sum=30.37$ & 41.63 \\
\hline & & $\mathrm{E}\left(\mathrm{S}^{2}\right)$ & 11.92 & $\mathrm{~V}\left(\mathrm{~S}^{2}\right)$ & 4.35 & & & $\chi^{2}(0.05,290)$ & $=124.3$ & & & & & $\chi^{2} 0.05,1=$ & 3.84 \\
\hline
\end{tabular}


Table.5 Non parametric measures of genotypes based on original values of yield 2016-17 (BLUE)

\begin{tabular}{|l|l|l|l|l|l|l|l|l|l|l|l|l|l|l|l|l|l|}
\hline blue16-17 & Yield & $\mathbf{G A I}$ & $\mathbf{M R}$ & $\mathbf{S D}$ & $\mathbf{C V}$ & $\mathbf{M e d}$ & $\mathbf{S}_{\mathbf{i}}^{\mathbf{1}}$ & $\mathbf{S}_{\mathbf{i}}^{\mathbf{2}}$ & $\mathbf{S}_{\mathbf{i}}^{\mathbf{3}}$ & $\mathbf{S}_{\mathbf{i}}^{\mathbf{4}}$ & $\mathbf{S}_{\mathbf{i}}^{\mathbf{5}}$ & $\mathbf{S}_{\mathbf{i}}^{\mathbf{6}}$ & $\mathbf{S}_{\mathbf{i}}^{\mathbf{7}}$ & $\mathbf{C M R}^{\mathbf{C}}$ & $\mathbf{C S D}^{\mathbf{C}}$ \\
\hline BRW3773 & 57.29 & 56.63 & 5.86 & 3.97 & 0.678 & 5 & 4.54 & 15.76 & 72.63 & 3.90 & 3.48 & 16.63 & 4.37 & 6.68 & 3.98 \\
\hline DBW189 & 55.41 & 54.71 & 7.14 & 3.56 & 0.498 & 8 & 4.08 & 12.65 & 47.80 & 3.49 & 3.06 & 12.00 & 3.98 & 6.64 & 3.76 \\
\hline DBW196 & 54.91 & 54.15 & 6.86 & 3.15 & 0.459 & 6.5 & 3.67 & 9.90 & 39.00 & 3.09 & 2.71 & 11.08 & 3.52 & 6.00 & 3.46 \\
\hline DBW88 & 54.88 & 54.33 & 7.86 & 2.99 & 0.381 & 9 & 3.37 & 8.94 & 30.73 & 2.94 & 2.47 & 8.80 & 3.49 & 6.75 & 3.36 \\
\hline HD2967 & 51.69 & 50.87 & 9.00 & 3.47 & 0.386 & 10.5 & 3.74 & 12.07 & 36.22 & 3.41 & 2.86 & 8.89 & 4.08 & 6.14 & 3.88 \\
\hline HD3086 & 57.05 & 56.13 & 5.39 & 3.35 & 0.621 & 5 & 3.90 & 11.21 & 56.13 & 3.29 & 2.88 & 14.94 & 3.76 & 6.57 & 3.75 \\
\hline HD3226 & 57.22 & 56.30 & 5.68 & 3.41 & 0.601 & 6.5 & 3.94 & 11.63 & 55.31 & 3.35 & 3.01 & 14.84 & 3.73 & 6.96 & 3.65 \\
\hline HP1963 & 56.94 & 56.29 & 5.75 & 2.85 & 0.496 & 5.5 & 3.26 & 8.12 & 38.13 & 2.80 & 2.32 & 11.30 & 3.37 & 6.57 & 2.79 \\
\hline PBW750 & 57.07 & 56.33 & 5.54 & 3.40 & 0.615 & 5.5 & 3.93 & 11.59 & 56.54 & 3.34 & 2.82 & 14.27 & 3.96 & 6.54 & 3.48 \\
\hline UP2942 & 55.82 & 55.11 & 6.79 & 3.36 & 0.495 & 7 & 3.89 & 11.29 & 44.91 & 3.30 & 2.76 & 11.39 & 3.94 & 6.75 & 3.56 \\
\hline WH1105 & 55.12 & 54.28 & 6.75 & 3.26 & 0.483 & 8 & 3.75 & 10.64 & 42.56 & 3.20 & 2.70 & 11.19 & 3.80 & 6.11 & 3.38 \\
\hline WH1202 & 57.10 & 56.50 & 5.14 & 2.92 & 0.567 & 5 & 3.26 & 8.50 & 44.61 & 2.86 & 2.24 & 12.22 & 3.65 & 6.29 & 2.76 \\
\hline
\end{tabular}

Table.6 Non parametric measures of genotypes based on corrected values of yield (BLUE)

\begin{tabular}{|c|c|c|c|c|c|c|c|c|c|c|c|c|c|c|c|}
\hline blue16-17 & $\mathrm{CCV}$ & Cmed & $\mathrm{CS}_{\mathrm{i}}{ }^{1}$ & $\mathrm{CS}_{\mathrm{i}}^{2}$ & $\mathrm{CS}_{\mathrm{i}}{ }^{3}$ & $\mathrm{CS}_{\mathrm{i}}{ }^{4}$ & $\mathrm{CS}_{\mathrm{i}}{ }^{5}$ & $\mathrm{CS}_{\mathrm{i}}{ }^{6}$ & $\mathrm{CS}_{\mathrm{i}}{ }^{7}$ & $\mathbf{N P}_{\mathbf{i}}^{(1)}$ & $\mathrm{NP}_{\mathbf{i}}{ }^{(2)}$ & $\mathbf{N P}_{\mathbf{i}}^{(3)}$ & $\mathrm{NP}_{\mathbf{i}}{ }^{(4)}$ & Z1 & $\mathbf{Z 2}$ \\
\hline BRW3773 & 0.596 & 6 & 4.61 & 15.86 & 64.10 & 3.91 & 3.51 & 16.39 & 4.35 & 3.46 & 0.693 & 0.668 & 0.787 & 3.20 & 3.57 \\
\hline DBW189 & 0.567 & 7 & 4.36 & 14.16 & 57.57 & 3.70 & 3.22 & 12.88 & 4.24 & 3.14 & 0.393 & 0.517 & 0.610 & 1.18 & 1.16 \\
\hline DBW196 & 0.577 & 6 & 4.03 & 12.00 & 54.00 & 3.40 & 2.86 & 13.33 & 4.05 & 2.86 & 0.440 & 0.496 & 0.588 & 0.03 & 0.00 \\
\hline DBW88 & 0.498 & 7 & 3.91 & 11.31 & 45.22 & 3.30 & 2.79 & 11.14 & 3.91 & 2.75 & 0.306 & 0.420 & 0.497 & 0.03 & 0.09 \\
\hline HD2967 & 0.631 & 5.5 & 4.50 & 15.02 & 66.00 & 3.81 & 3.44 & 17.51 & 4.21 & 3.43 & 0.327 & 0.423 & 0.500 & 2.21 & 2.21 \\
\hline HD3086 & 0.570 & 6 & 4.37 & 14.03 & 57.65 & 3.68 & 3.29 & 15.33 & 4.12 & 3.29 & 0.657 & 0.682 & 0.809 & 1.21 & 1.03 \\
\hline HD3226 & 0.524 & 8 & 4.21 & 13.29 & 51.54 & 3.58 & 3.26 & 11.39 & 3.94 & 3.11 & 0.478 & 0.631 & 0.741 & 0.44 & 0.44 \\
\hline HP1963 & 0.425 & 6.5 & 3.23 & 7.81 & 32.09 & 2.74 & 2.29 & 9.85 & 3.29 & 2.29 & 0.416 & 0.477 & 0.562 & 4.29 & 3.88 \\
\hline PBW750 & 0.532 & 6 & 4.03 & 12.11 & 50.03 & 3.42 & 3.00 & 14.01 & 3.89 & 2.96 & 0.539 & 0.617 & 0.728 & 0.03 & 0.01 \\
\hline UP2942 & 0.527 & 7 & 4.13 & 12.64 & 50.56 & 3.49 & 3.00 & 12.00 & 4.06 & 2.96 & 0.423 & 0.514 & 0.609 & 0.19 & 0.12 \\
\hline WH1105 & 0.554 & 6 & 3.90 & 11.43 & 50.54 & 3.32 & 2.77 & 12.90 & 3.99 & 2.75 & 0.344 & 0.492 & 0.578 & 0.04 & 0.05 \\
\hline \multirow[t]{3}{*}{ WH1202 } & 0.439 & 6 & 3.17 & 7.62 & 32.73 & 2.71 & 2.26 & 10.52 & 3.26 & 2.21 & 0.443 & 0.527 & 0.616 & 5.05 & 4.25 \\
\hline & & $\mathrm{E}\left(\mathrm{S}^{1}\right)$ & 3.97 & $\mathrm{~V}\left(\mathrm{~S}^{1}\right)$ & 0.1276 & $\mathrm{~W}=$ & 0.296 & $\chi^{2}=$ & 97.600 & & & & & $\sum=17.89$ & 16.80 \\
\hline & & $\mathrm{E}\left(\mathrm{S}^{2}\right)$ & 11.92 & $\mathrm{~V}\left(\mathrm{~S}^{2}\right)$ & 4.35 & & & $\chi^{2}(0.05,290)$ & $=124.3$ & & & & & $\chi^{2}(0.05,1)$ & $=3.84$ \\
\hline
\end{tabular}


Table.7 Non parametric measures of genotypes based on original values of yield 2017-18 (BLUP)

\begin{tabular}{|c|c|c|c|c|c|c|c|c|c|c|c|c|c|c|c|}
\hline blup 17-18 & Yield & GAI & MR & SD & CV & Med & $S_{i}{ }^{1}$ & $\mathrm{~S}_{\mathrm{i}}^{2}$ & $\mathbf{S}_{\mathrm{i}}{ }^{3}$ & $S_{i}{ }^{4}$ & $S_{i}{ }^{5}$ & $S_{i}^{6}$ & $\mathrm{~S}_{\mathrm{i}}^{7}$ & CMR & CSD \\
\hline UP 2981 & 55.30 & 54.60 & 8.36 & 4.39 & 0.5247 & 8 & 5.08 & 19.24 & 55.23 & 4.30 & 3.57 & 10.69 & 5.17 & 8.60 & 4.05 \\
\hline DBW 221 & 56.72 & 56.17 & 6.24 & 4.55 & 0.7289 & 4 & 5.11 & 25.37 & 97.58 & 4.94 & 4.53 & 18.15 & 5.38 & 8.32 & 4.56 \\
\hline DBW 222 & 54.53 & 53.82 & 9.00 & 4.77 & 0.5300 & 9 & 5.55 & 23.18 & 61.80 & 4.72 & 4.03 & 11.18 & 5.53 & 8.24 & 4.52 \\
\hline BRW 3792 & 58.00 & 57.19 & 5.04 & 5.06 & 1.0044 & 3 & 5.25 & 37.11 & 176.69 & 5.97 & 5.71 & 28.31 & 6.24 & 7.84 & 4.76 \\
\hline PBW 763 & 52.14 & 51.49 & 11.68 & 4.36 & 0.3730 & 12 & 4.81 & 30.46 & 62.59 & 5.41 & 5.01 & 10.73 & 5.83 & 8.08 & 4.88 \\
\hline PBW 766 & 56.09 & 55.26 & 6.80 & 4.41 & 0.6480 & 6 & 5.06 & 21.95 & 77.48 & 4.59 & 4.18 & 15.37 & 5.04 & 8.00 & 4.38 \\
\hline DBW 233 & 56.34 & 55.37 & 6.80 & 5.09 & 0.7487 & 5 & 5.83 & 28.45 & 100.42 & 5.23 & 4.77 & 17.54 & 5.73 & 8.08 & 5.12 \\
\hline HD 3226 & 55.94 & 55.25 & 7.04 & 4.51 & 0.6411 & 6 & 5.16 & 22.19 & 75.64 & 4.62 & 4.26 & 15.13 & 5.00 & 8.52 & 3.97 \\
\hline PBW 801 & 54.33 & 53.67 & 8.64 & 3.57 & 0.4131 & 8 & 4.14 & 12.82 & 35.62 & 3.51 & 2.97 & 8.61 & 4.14 & 7.60 & 3.86 \\
\hline PBW 800 & 55.25 & 54.66 & 7.44 & 4.80 & 0.6447 & 8 & 5.53 & 23.89 & 77.06 & 4.79 & 4.12 & 13.85 & 5.56 & 7.92 & 4.97 \\
\hline DPW 621-50 & 53.27 & 52.69 & 10.48 & 4.37 & 0.4169 & 11 & 5.03 & 23.78 & 54.45 & 4.78 & 4.19 & 10.00 & 5.45 & 7.80 & 4.13 \\
\hline HD 3086 & 53.55 & 53.08 & 9.68 & 4.52 & 0.4665 & 10 & 5.27 & 22.21 & 55.06 & 4.62 & 3.88 & 10.01 & 5.50 & 7.56 & 4.61 \\
\hline HD 2967 & 54.03 & 53.31 & 9.16 & 4.22 & 0.4607 & 9 & 4.90 & 18.47 & 48.40 & 4.21 & 3.49 & 9.52 & 5.08 & 7.44 & 3.79 \\
\hline DBW 88 & 53.23 & 52.71 & 10.72 & 4.08 & 0.3804 & 12 & 4.44 & 22.43 & 50.21 & 4.64 & 4.24 & 9.89 & 5.08 & 8.32 & 3.78 \\
\hline WH 1105 & 53.14 & 52.58 & 10.44 & 4.08 & 0.3911 & 11 & 4.73 & 21.18 & 48.69 & 4.51 & 3.94 & 9.43 & 5.16 & 7.68 & 4.23 \\
\hline
\end{tabular}

Table.8 Non parametric measures of genotypes based on corrected values of yield (BLUP)

\begin{tabular}{|c|c|c|c|c|c|c|c|c|c|c|c|c|c|c|c|}
\hline & CCV & Cmed & $\mathrm{CS}_{\mathbf{i}}{ }^{1}$ & $\mathrm{CS}_{\mathrm{i}}{ }^{2}$ & $\mathrm{CS}_{\mathrm{i}}{ }^{3}$ & $\mathrm{CS}_{\mathrm{i}}{ }^{4}$ & $\mathrm{CS}_{\mathrm{i}}^{5}$ & $\mathrm{CS}_{\mathrm{i}}{ }^{6}$ & $\mathrm{CS}_{\mathrm{i}}{ }^{\mathrm{T}}$ & $\mathrm{NP}_{\mathbf{i}}{ }^{(1)}$ & $\mathbf{N P}_{\mathbf{i}}{ }^{(2)}$ & $\mathbf{N P}_{\mathbf{i}}{ }^{(3)}$ & $\mathbf{N P}_{\mathbf{i}}{ }^{(4)}$ & Z1 & $\mathbf{Z 2}$ \\
\hline UP 2981 & 0.4711 & 8.0 & 4.69 & 16.42 & 45.81 & 3.97 & 3.34 & 10.45 & 4.71 & 3.32 & 0.4150 & 0.4749 & 0.5614 & 0.3529 & 0.416 \\
\hline DBW 221 & 0.5483 & 8.0 & 5.28 & 20.89 & 60.26 & 4.48 & 4.10 & 12.83 & 4.89 & 4.08 & 1.0200 & 0.7177 & 0.8462 & 0.3984 & 0.407 \\
\hline DBW 222 & 0.5487 & 8.0 & 5.25 & 20.58 & 59.93 & 4.44 & 3.78 & 11.83 & 5.22 & 3.76 & 0.4178 & 0.4938 & 0.5830 & 0.3154 & 0.299 \\
\hline BRW 3792 & 0.6069 & 7.0 & 5.52 & 23.24 & 71.15 & 4.72 & 3.99 & 14.26 & 5.59 & 3.80 & 1.2667 & 0.9372 & 1.0952 & 1.2823 & 1.721 \\
\hline PBW 763 & 0.6041 & 8.0 & 5.70 & 24.11 & 71.61 & 4.81 & 4.23 & 13.23 & 5.47 & 4.16 & 0.3467 & 0.4119 & 0.4880 & 2.2750 & 2.435 \\
\hline PBW 766 & 0.5472 & 8.0 & 5.11 & 19.54 & 58.63 & 4.33 & 3.86 & 12.08 & 4.86 & 3.84 & 0.6400 & 0.6370 & 0.7510 & 0.0725 & 0.063 \\
\hline DBW 233 & 0.6340 & 8.0 & 5.93 & 26.53 & 78.79 & 5.05 & 4.58 & 14.33 & 5.55 & 4.56 & 0.9120 & 0.7421 & 0.8716 & 3.9272 & 5.077 \\
\hline HD 3226 & 0.4659 & 9.0 & 4.62 & 15.77 & 44.41 & 3.89 & 3.22 & 8.93 & 4.71 & 3.20 & 0.5333 & 0.5526 & 0.6563 & 0.5583 & 0.691 \\
\hline PBW 801 & 0.5082 & 7.0 & 4.43 & 15.96 & 50.39 & 3.91 & 3.29 & 11.74 & 4.66 & 3.00 & 0.3750 & 0.4530 & 0.5131 & 1.2929 & 0.603 \\
\hline PBW 800 & 0.6281 & 10.0 & 5.75 & 25.23 & 76.44 & 4.92 & 4.33 & 10.82 & 5.60 & 4.24 & 0.5300 & 0.6614 & 0.7724 & 2.5785 & 3.536 \\
\hline DPW 621-50 & 0.5299 & 7.0 & 4.81 & 17.75 & 54.62 & 4.13 & 3.68 & 13.14 & 4.63 & 3.52 & 0.3200 & 0.3939 & 0.4593 & 0.1179 & 0.069 \\
\hline HD 3086 & 0.6099 & 7.0 & 5.37 & 22.38 & 71.06 & 4.64 & 3.95 & 14.11 & 5.44 & 3.84 & 0.3840 & 0.4789 & 0.5544 & 0.6596 & 1.136 \\
\hline HD 2967 & 0.5090 & 7.0 & 4.39 & 15.74 & 50.78 & 3.89 & 3.24 & 11.57 & 4.66 & 3.08 & 0.3422 & 0.4244 & 0.4796 & 1.4898 & 0.703 \\
\hline DBW 88 & 0.4547 & 10.0 & 4.31 & 14.39 & 41.51 & 3.72 & 3.24 & 8.10 & 4.26 & 3.04 & 0.2533 & 0.3467 & 0.4017 & 1.9644 & 1.503 \\
\hline \multirow[t]{3}{*}{ WH 1105} & 0.5508 & 7.0 & 4.96 & 18.78 & 58.67 & 4.25 & 3.75 & 13.40 & 4.80 & 3.64 & 0.3309 & 0.4067 & 0.4751 & 0.0014 & 0.001 \\
\hline & & $\mathrm{E}\left(\mathrm{S}^{1}\right)$ & 4.98 & $\mathrm{~V}\left(\mathrm{~S}^{1}\right)$ & 0.2293 & & & $\mathrm{~W}=$ & 0.3091 & $\chi^{2}=129.82$ & & & & $\sum=17.29$ & 18.66 \\
\hline & & $\mathrm{E}\left(\mathrm{S}^{2}\right)$ & 18.67 & $V\left(S^{2}\right)$ & 12.1624 & & & & $\chi^{2}(0.05,290)$ & $=124.3$ & & & & $\chi^{2}(0.05,1)=$ & 3.84 \\
\hline
\end{tabular}


Table.9 Non parametric measures of genotypes based on original values of yield 2017-18 (BLUE)

\begin{tabular}{|c|c|c|c|c|c|c|c|c|c|c|c|c|c|c|c|}
\hline blue 17-18 & Yield & GAI & MR & SD & CV & Med & $S_{i}{ }^{1}$ & $S_{i}^{2}$ & $\mathbf{S i}_{\mathrm{i}}{ }^{3}$ & $S_{i}{ }^{4}$ & $S_{i}^{5}$ & $S_{i}^{6}$ & $S_{i}^{7}$ & CMR & CSD \\
\hline UP 2981 & 55.18 & 54.42 & 8.00 & 4.20 & 0.5254 & 8 & 4.91 & 4.93 & 53.00 & 4.12 & 3.44 & 10.75 & 17.67 & 8.32 & 4.38 \\
\hline DBW 221 & 56.85 & 56.28 & 5.84 & 4.20 & 0.7192 & 3 & 4.73 & 4.53 & 72.49 & 4.12 & 3.74 & 16.01 & 17.64 & 7.92 & 4.62 \\
\hline DBW 222 & 54.49 & 53.71 & 8.44 & 4.65 & 0.5505 & 8 & 5.39 & 5.11 & 61.39 & 4.55 & 4.06 & 12.02 & 21.59 & 7.92 & 4.70 \\
\hline BRW 3792 & 58.13 & 57.20 & 5.08 & 4.78 & 0.9405 & 3 & 4.99 & 5.76 & 107.84 & 4.68 & 3.80 & 18.71 & 22.83 & 7.84 & 4.76 \\
\hline PBW 763 & 52.07 & 51.36 & 10.52 & 3.90 & 0.3703 & 11 & 4.47 & 4.53 & 34.62 & 3.82 & 3.22 & 7.64 & 15.18 & 8.32 & 4.60 \\
\hline PBW 766 & 56.19 & 55.32 & 6.36 & 3.85 & 0.6054 & 6 & 4.43 & 4.47 & 55.94 & 3.77 & 3.18 & 12.50 & 14.82 & 8.00 & 4.11 \\
\hline DBW 233 & 56.31 & 55.28 & 6.88 & 4.65 & 0.6757 & 5 & 5.34 & 5.05 & 75.38 & 4.55 & 4.11 & 14.92 & 21.61 & 8.08 & 4.82 \\
\hline HD 3226 & 56.08 & 55.42 & 6.84 & 4.03 & 0.5889 & 6 & 4.69 & 4.55 & 56.92 & 3.95 & 3.42 & 12.50 & 16.22 & 8.00 & 4.36 \\
\hline PBW 801 & 54.36 & 53.69 & 8.04 & 3.45 & 0.4286 & 7 & 3.93 & 4.12 & 35.44 & 3.38 & 2.77 & 8.61 & 11.87 & 7.76 & 4.11 \\
\hline PBW 800 & 55.22 & 54.61 & 7.16 & 4.52 & 0.6307 & 8 & 5.25 & 5.11 & 68.35 & 4.42 & 3.83 & 13.39 & 20.39 & 7.80 & 4.84 \\
\hline DPW 621-50 & 53.30 & 52.68 & 9.48 & 3.91 & 0.4121 & 10 & 4.51 & 4.49 & 38.63 & 3.83 & 3.26 & 8.60 & 15.26 & 7.88 & 4.38 \\
\hline HD 3086 & 53.58 & 53.11 & 9.04 & 3.93 & 0.4349 & 8 & 4.52 & 4.69 & 41.04 & 3.85 & 3.16 & 8.75 & 15.46 & 7.72 & 4.37 \\
\hline HD 2967 & 53.90 & 53.10 & 8.72 & 4.13 & 0.4734 & 9 & 4.81 & 4.88 & 46.91 & 4.04 & 3.35 & 9.61 & 17.04 & 7.80 & 4.01 \\
\hline DBW 88 & 53.22 & 52.68 & 9.80 & 3.51 & 0.3584 & 11 & 3.93 & 4.20 & 30.20 & 3.44 & 2.82 & 7.18 & 12.33 & 8.52 & 3.53 \\
\hline WH 1105 & 52.99 & 52.36 & 9.48 & 3.95 & 0.4165 & 9 & 4.53 & 4.49 & 39.48 & 3.87 & 3.34 & 8.80 & 15.59 & 8.12 & 4.27 \\
\hline
\end{tabular}

Table.10 Non parametric measures of genotypes based on corrected values of yield (BLUE)

\begin{tabular}{|c|c|c|c|c|c|c|c|c|c|c|c|c|c|c|c|}
\hline & CCV & Cmed & $\mathrm{CS}_{\mathrm{i}}{ }^{1}$ & $\mathrm{CS}_{\mathrm{i}}^{2}$ & $\mathrm{CS}_{\mathrm{i}}{ }^{3}$ & $\mathrm{CS}_{\mathrm{i}}{ }^{4}$ & $\mathrm{CS}_{\mathrm{i}}^{5}$ & $\mathrm{CS}_{\mathrm{i}}^{6}$ & $\mathbf{C S}_{\mathbf{i}}^{7}$ & $\mathbf{N P}_{\mathbf{i}}^{\left({ }^{(1)}\right.}$ & $\mathbf{N P}_{\mathrm{i}}^{(2)}$ & $\mathbf{N P}_{i}{ }^{(3)}$ & $\mathbf{N P}_{\mathbf{i}}^{(4)}$ & $\mathrm{Z1}$ & $\mathbf{Z 2}$ \\
\hline UP 2981 & 0.5270 & 9 & 5.12 & 19.23 & 55.46 & 4.30 & 3.75 & 10.41 & 4.93 & 3.72 & 0.4650 & 0.5370 & 0.6400 & 0.0882 & 0.026 \\
\hline DBW 221 & 0.5831 & 7 & 5.33 & 21.33 & 64.63 & 4.52 & 4.16 & 14.85 & 4.93 & 4.12 & 1.3733 & 0.7748 & 0.9132 & 0.5514 & 0.582 \\
\hline DBW 222 & 0.5933 & 7 & 5.46 & 22.08 & 66.90 & 4.60 & 4.08 & 14.56 & 5.20 & 4.04 & 0.5050 & 0.5455 & 0.6469 & 1.0142 & 0.956 \\
\hline BRW 3792 & 0.6069 & 8 & 5.51 & 22.64 & 69.31 & 4.66 & 3.78 & 11.81 & 5.75 & 3.76 & 1.2533 & 0.9177 & 1.0853 & 1.2510 & 1.298 \\
\hline PBW 763 & 0.5527 & 8 & 5.34 & 21.14 & 60.99 & 4.51 & 3.72 & 11.62 & 5.46 & 3.68 & 0.3345 & 0.4283 & 0.5076 & 0.5723 & 0.504 \\
\hline PBW 766 & 0.5141 & 8 & 4.81 & 16.92 & 50.75 & 4.03 & 3.60 & 11.25 & 4.51 & 3.60 & 0.6000 & 0.6336 & 0.7568 & 0.1179 & 0.252 \\
\hline DBW 233 & 0.5967 & 8 & 5.55 & 23.24 & 69.04 & 4.72 & 4.33 & 13.53 & 5.15 & 4.32 & 0.8640 & 0.6866 & 0.8072 & 1.4448 & 1.722 \\
\hline HD 3226 & 0.5449 & 8 & 5.08 & 19.00 & 57.00 & 4.27 & 3.60 & 11.25 & 5.07 & 3.60 & 0.6000 & 0.6244 & 0.7427 & 0.0456 & 0.009 \\
\hline PBW 801 & 0.5291 & 7 & 4.73 & 16.86 & 52.13 & 4.02 & 3.31 & 11.83 & 4.89 & 3.16 & 0.4514 & 0.5003 & 0.5879 & 0.2750 & 0.269 \\
\hline PBW 800 & 0.6204 & 10 & 5.57 & 23.42 & 72.05 & 4.74 & 4.30 & 10.76 & 5.22 & 4.12 & 0.5150 & 0.6622 & 0.7784 & 1.5470 & 1.855 \\
\hline DPW 621-50 & 0.5560 & 6 & 5.10 & 19.19 & 58.46 & 4.29 & 3.88 & 16.15 & 4.75 & 3.80 & 0.3800 & 0.4528 & 0.5380 & 0.0652 & 0.023 \\
\hline HD 3086 & 0.5665 & 7 & 5.07 & 19.13 & 59.46 & 4.29 & 3.63 & 12.96 & 5.06 & 3.60 & 0.4500 & 0.4740 & 0.5612 & 0.0398 & 0.017 \\
\hline HD 2967 & 0.5142 & 8 & 4.63 & 16.08 & 49.49 & 3.93 & 3.20 & 10.00 & 4.83 & 3.16 & 0.3511 & 0.4506 & 0.5306 & 0.5377 & 0.549 \\
\hline DBW 88 & 0.4137 & 9 & 4.04 & 12.43 & 35.00 & 3.45 & 2.90 & 8.04 & 4.12 & 2.80 & 0.2545 & 0.3524 & 0.4122 & 3.8357 & 3.201 \\
\hline \multirow[t]{3}{*}{ WH 1105} & 0.5253 & 7 & 4.98 & 18.19 & 53.77 & 4.18 & 3.73 & 13.34 & 4.68 & 3.68 & 0.4089 & 0.4408 & 0.5253 & 0.0000 & 0.018 \\
\hline & & & $\mathrm{E}\left(\mathrm{S}^{1}\right)$ & 4.98 & $\mathrm{~V}\left(\mathrm{~S}^{1}\right)$ & 0.2293 & & $\mathrm{~W}=$ & 0.3287 & $\chi^{2}=138.06$ & & & & $\sum=11.39$ & 11.28 \\
\hline & & & $\mathrm{E}\left(\mathrm{S}^{2}\right)$ & 18.67 & $\mathrm{~V}\left(\mathrm{~S}^{2}\right)$ & 12.1624 & & & & $\chi^{2}(0.05,290)$ & $=124.3$ & & & $\chi^{2}(0.05,1)=$ & 3.84 \\
\hline
\end{tabular}


Table.11 Spearman's rank correlation analysis among non parametric measures (2016-17) BLUP

\begin{tabular}{|c|c|c|c|c|c|c|c|c|c|c|c|c|c|c|c|c|c|c|c|c|c|c|c|c|c|c|c|c|c|}
\hline & GAI & MR & SD & CV & Med & $\mathbf{S}_{\mathrm{i}}{ }^{1}$ & $S_{i}^{2}$ & $\mathbf{S}_{\mathrm{i}}^{3}$ & $S_{i}^{4}$ & $\mathbf{S}_{\mathrm{i}}^{5}$ & $\mathbf{S}_{\mathrm{i}}^{6}$ & $S_{i}^{7}$ & CMR & CSD & CCV & Cmed & $\mathbf{C S}_{\mathbf{i}}^{1}$ & $\mathrm{CS}_{\mathrm{i}}^{2}$ & $\mathbf{C S}_{i}^{3}$ & $\mathbf{C S}_{i}{ }^{4}$ & $\mathbf{C S}_{\mathrm{i}}^{5}$ & $\mathbf{C S}_{\mathrm{i}}^{6}$ & $\mathbf{C S}_{\mathrm{i}}^{7}$ & $\mathbf{N P}_{\mathbf{i}}{ }^{(1)}$ & $\mathbf{N P}_{\mathbf{i}}^{(2)}$ & $\mathbf{N P}_{\mathbf{i}}^{(3)}$ & $\mathbf{N P}_{\mathbf{i}}^{(4)}$ & Z1 & $\mathbf{Z 2}$ \\
\hline Yield & 0.9720 & -0.9126 & -0.3217 & -0.8392 & 0.8811 & -0.3636 & -0.3706 & -0.7203 & -0.3217 & -0.3077 & -0.8182 & -0.3217 & 0.4196 & -0.1469 & -0.0559 & -0.2343 & -0.1818 & -0.1538 & -0.1748 & -0.1014 & -0.1853 & -0.0350 & -0.1014 & -0.1853 & -0.8182 & -0.8112 & -0.8112 & 0.1189 & 0.1538 \\
\hline GAI & 1.0000 & -0.8846 & -0.3077 & -0.8042 & 0.8531 & -0.3217 & \begin{tabular}{|l|l|}
-0.3916 \\
\end{tabular} & -0.7063 & -0.3077 & -0.2657 & -0.7902 & -0.3077 & 0.4196 & -0.0490 & 0.0280 & -0.2343 & -0.1049 & -0.0559 & -0.0769 & 0.0035 & -0.1224 & 0.0350 & 0.0035 & -0.1224 & -0.7832 & -0.7622 & -0.7622 & 0.0769 & 0.0559 \\
\hline MR & & 1.0000 & 0.1154 & 0.7587 & -0.8776 & 0.1573 & 0.2203 & 0.6049 & 0.1154 & 0.0594 & 0.7378 & 0.1154 & -0.0035 & -0.1014 & 0.0385 & -0.2098 & -0.0664 & -0.0315 & 0.0385 & -0.0769 & \begin{tabular}{|l|l|}
-0.0070 \\
\end{tabular} & 0.0105 & -0.0769 & -0.0070 & 0.7028 & 0.7587 & 0.7587 & -0.1713 & -0.0105 \\
\hline SD & & & 1.0000 & 0.6294 & \begin{tabular}{|l}
-0.1399 \\
\end{tabular} & 0.9720 & 0.9161 & 0.8252 & 1.0000 & 0.9441 & 0.6364 & 1.0000 & $\begin{array}{l}-0.1049 \\
\end{array}$ & 0.8671 & 0.7483 & 0.0874 & 0.9021 & 0.8601 & 0.8671 & 0.8566 & 0.8986 & 0.8322 & 0.8566 & 0.8986 & 0.6434 & 0.6643 & 0.6643 & -0.0070 & -0.8601 \\
\hline CV & & & & 1.0000 & -0.7343 & 0.6993 & 0.5944 & 0.9301 & 0.6294 & 0.6084 & 0.9930 & 0.6294 & -0.2448 & 0.4615 & 0.3566 & 0.1364 & 0.5105 & 0.3986 & 0.4615 & 0.4510 & 0.5629 & 0.4056 & 0.4510 & 0.5629 & 0.9510 & 0.9720 & 0.9720 & -0.2517 & -0.3986 \\
\hline Med & & & & & 1.0000 & -0.1888 & \begin{tabular}{|l|l|} 
& -0.1958 \\
\end{tabular} & $\begin{array}{l}-0.6014 \\
\end{array}$ & -0.1399 & -0.1119 & -0.7203 & $\begin{array}{l}-0.1399 \\
\end{array}$ & 0.1538 & 0.0280 & -0.0420 & 0.1643 & -0.0070 & 0.0070 & -0.0629 & \begin{tabular}{|l|l|} 
\\
\end{tabular} .0734 & -0.0175 & -0.0490 & 0.0734 & -0.0175 & -0.6993 & -0.7133 & -0.7133 & 0.3287 & 0.1049 \\
\hline$S_{i}{ }^{1}$ & & & & & & 1.0000 & 0.8252 & 0.8392 & 0.9720 & 0.9650 & 0.7133 & 0.9720 & -0.1469 & 0.8951 & 0.7343 & 0.1643 & 0.9231 & 0.8182 & 0.8741 & 0.8846 & 0.9266 & 0.7972 & 0.8846 & 0.9266 & 0.6923 & 0.7133 & 0.7133 & $\begin{array}{l}-0.06999 \\
\end{array}$ & -0.8182 \\
\hline $\mathbf{S}_{\mathrm{i}}^{2}$ & & & & & & & 1.0000 & 0.7902 & 0.9161 & 0.7762 & 0.5734 & 0.9161 & -0.0839 & 0.6993 & 0.6783 & -0.0385 & 0.7483 & 0.8112 & 0.7133 & 0.6888 & 0.7238 & 0.7413 & 0.6888 & 0.7238 & 0.6014 & 0.6573 & 0.6573 & 0.1329 & -0.8112 \\
\hline $\mathbf{S}_{\mathbf{i}}^{3}$ & & & & & & & & 1.0000 & 0.8252 & 0.7902 & 0.9371 & 0.8252 & -0.1678 & 0.6294 & 0.5734 & 0.0594 & 0.6783 & 0.6014 & 0.6713 & 0.6189 & 0.7238 & 0.6294 & 0.6189 & 0.7238 & 0.9301 & 0.9441 & 0.9441 & -0.1189 & -0.6014 \\
\hline$S_{i}^{4}$ & & & & & & & & & 1.0000 & 0.9441 & 0.6364 & 1.0000 & -0.1049 & 0.8671 & 0.7483 & 0.0874 & 0.9021 & 0.8601 & 0.8671 & 0.8566 & 0.8986 & 0.8322 & 0.8566 & 0.8986 & 0.6434 & 0.6643 & 0.6643 & -0.0070 & -0.8601 \\
\hline $\mathbf{S}_{i}^{5}$ & & & & & & & & & & 1.0000 & 0.6364 & 0.9441 & -0.2168 & 0.9371 & 0.7133 & 0.2483 & 0.9580 & 0.8182 & 0.8951 & 0.9266 & 0.9685 & 0.7622 & 0.9266 & 0.9685 & 0.6573 & 0.6364 & 0.6364 & -0.0210 & -0.8182 \\
\hline$S_{i}{ }^{6}$ & & & & & & & & & & & 1.0000 & 0.6364 & -0.2378 & 0.4685 & 0.3636 & 0.1364 & 0.5175 & 0.3776 & 0.4755 & 0.4580 & 0.5769 & 0.4126 & 0.4580 & 0.5769 & 0.9441 & 0.9580 & 0.9580 & -0.2587 & $\mid-0.3776$ \\
\hline $\mathbf{S}_{\mathrm{i}}^{7}$ & & & & & & & & & & & & 1.0000 & -0.1049 & 0.8671 & 0.7483 & 0.0874 & 0.9021 & 0.8601 & 0.8671 & 0.8566 & 0.8986 & 0.8322 & 0.8566 & 0.8986 & 0.6434 & 0.6643 & 0.6643 & -0.0070 & -0.8601 \\
\hline CMR & & & & & & & & & & & & & 1.0000 & -0.2028 & 0.3427 & -0.8217 & -0.2168 & -0.0350 & 0.1119 & -0.1364 & -0.1853 & 0.3077 & -0.1364 & -0.1853 & -0.2517 & -0.1678 & -0.1678 & 0.0490 & 0.0769 \\
\hline CSD & & & & & & & & & & & & & & 1.0000 & 0.7483 & 0.2413 & 0.9930 & 0.9091 & 0.9161 & 0.9965 & 0.9685 & 0.7902 & 0.9965 & 0.9685 & 0.5175 & 0.5105 & 0.5105 & -0.0490 & -0.9091 \\
\hline CCV & & & & & & & & & & & & & & & 1.0000 & -0.3182 & 0.7273 & 0.8322 & 0.9161 & 0.7797 & 0.7028 & 0.9231 & 0.7797 & 0.7028 & 0.3776 & 0.4615 & 0.4615 & 0.1608 & -0.8322 \\
\hline Cmed & & & & & & & & & & & & & & & & 1.0000 & 0.2552 & -0.0245 & -0.0524 & 0.2378 & 0.3287 & -0.3392 & 0.2378 & 0.3287 & 0.2133 & 0.0664 & 0.0664 & 0.0664 & 0.0245 \\
\hline $\mathbf{C S}_{\mathrm{i}}{ }^{1}$ & & & & & & & & & & & & & & & & & 1.0000 & 0.8951 & 0.9021 & 0.9825 & 0.9825 & 0.7832 & 0.9825 & 0.9825 & 0.5664 & 0.5524 & 0.5524 & -0.0559 & -0.8951 \\
\hline $\mathbf{C S}_{i}{ }^{2}$ & & & & & & & & & & & & & & & & & & 1.0000 & 0.9091 & 0.9196 & 0.8357 & 0.8811 & 0.9196 & 0.8357 & 0.4336 & 0.4825 & 0.4825 & 0.0000 & -1.0000 \\
\hline $\mathrm{CS}_{\mathrm{i}}{ }^{3}$ & & & & & & & & & & & & & & & & & & & 1.0000 & 0.9336 & 0.8916 & 0.9091 & 0.9336 & 0.8916 & 0.5245 & 0.5455 & 0.5455 & 0.0280 & -0.9091 \\
\hline $\mathrm{CS}_{i}{ }^{4}$ & & & & & & & & & & & & & & & & & & & & 1.0000 & 0.9580 & 0.8077 & 1.0000 & 0.9580 & 0.5070 & 0.5070 & 0.5070 & 0.0035 & -0.8636 \\
\hline $\mathrm{CS}_{1}^{5}$ & & & & & & & & & & & & & & & & & & & & & 1.0000 & 0.7587 & 0.9580 & 1.0000 & 0.6469 & 0.6049 & 0.6049 & -0.0175 & -0.7797 \\
\hline $\mathbf{C S}_{\mathrm{i}}{ }^{6}$ & & & & & & & & & & & & & & & & & & & & & & 1.0000 & 0.8077 & 0.7587 & 0.4126 & 0.4825 & 0.4825 & -0.1049 & -0.8811 \\
\hline $\mathbf{C S}_{i}^{7}$ & & & & & & & & & & & & & & & & & & & & & & & 1.0000 & 0.9580 & 0.5070 & 0.5070 & 0.5070 & 0.0035 & -0.8636 \\
\hline $\mathbf{N P}_{\mathrm{i}}{ }^{(1)}$ & & & & & & & & & & & & & & & & & & & & & & & & 1.0000 & 0.6469 & 0.6049 & 0.6049 & -0.0175 & -0.7797 \\
\hline $\mathbf{N P}_{\mathrm{i}}{ }^{(2)}$ & & & & & & & & & & & & & & & & & & & & & & & & & 1.0000 & 0.9650 & 0.9650 & -0.1538 & -0.4336 \\
\hline $\mathbf{N} \mathbf{P}_{i}{ }^{(3)}$ & & & & & & & & & & & & & & & & & & & & & & & & & & 1.0000 & 1.0000 & -0.0979 & -0.4825 \\
\hline $\mathbf{N P}_{\mathbf{i}}{ }^{(4)}$ & & & & & & & & & & & & & & & & & & & & & & & & & & & 1.0000 & -0.0979 & -0.4825 \\
\hline $\mathrm{Z1}$ & & & & & & & & & & & & & & & & & & & & & & & & & & & & 1.0000 & -0.5350 \\
\hline
\end{tabular}




\section{Int.J.Curr.Microbiol.App.Sci (2020) 9(7): 1167-1190}

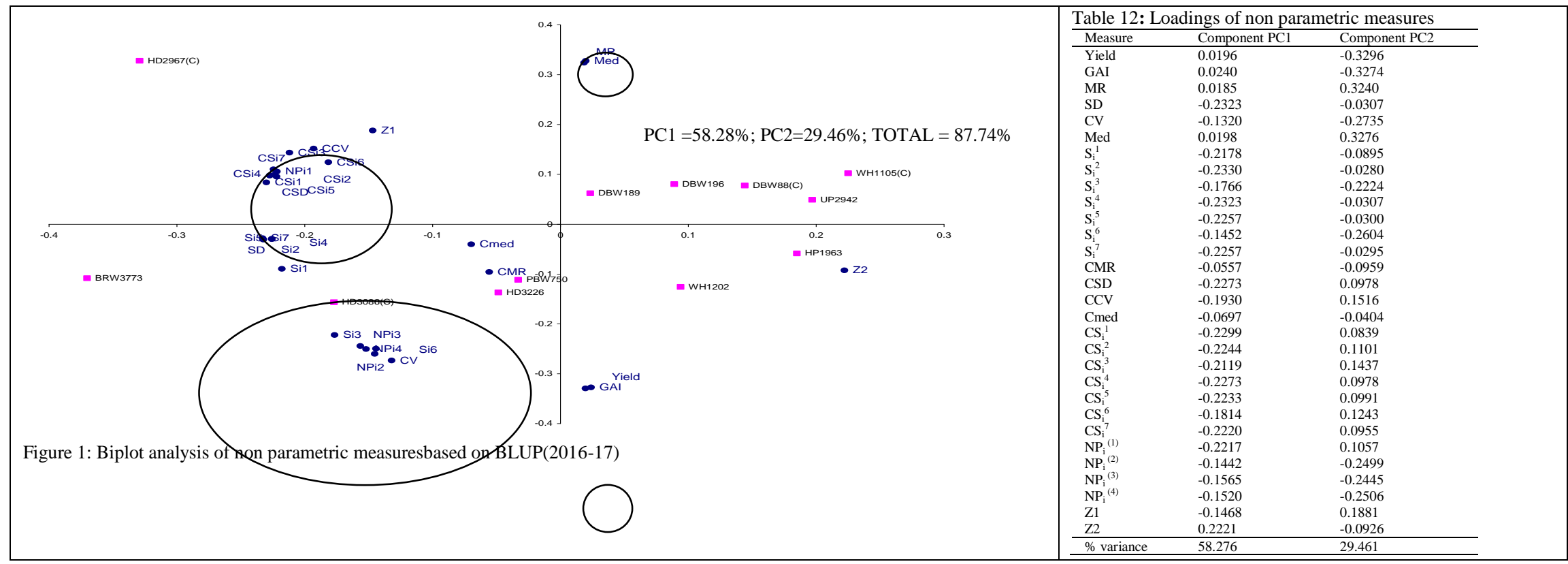


Table.13 Spearman's rank correlation values among non parametric measures (2016-17) BLUE

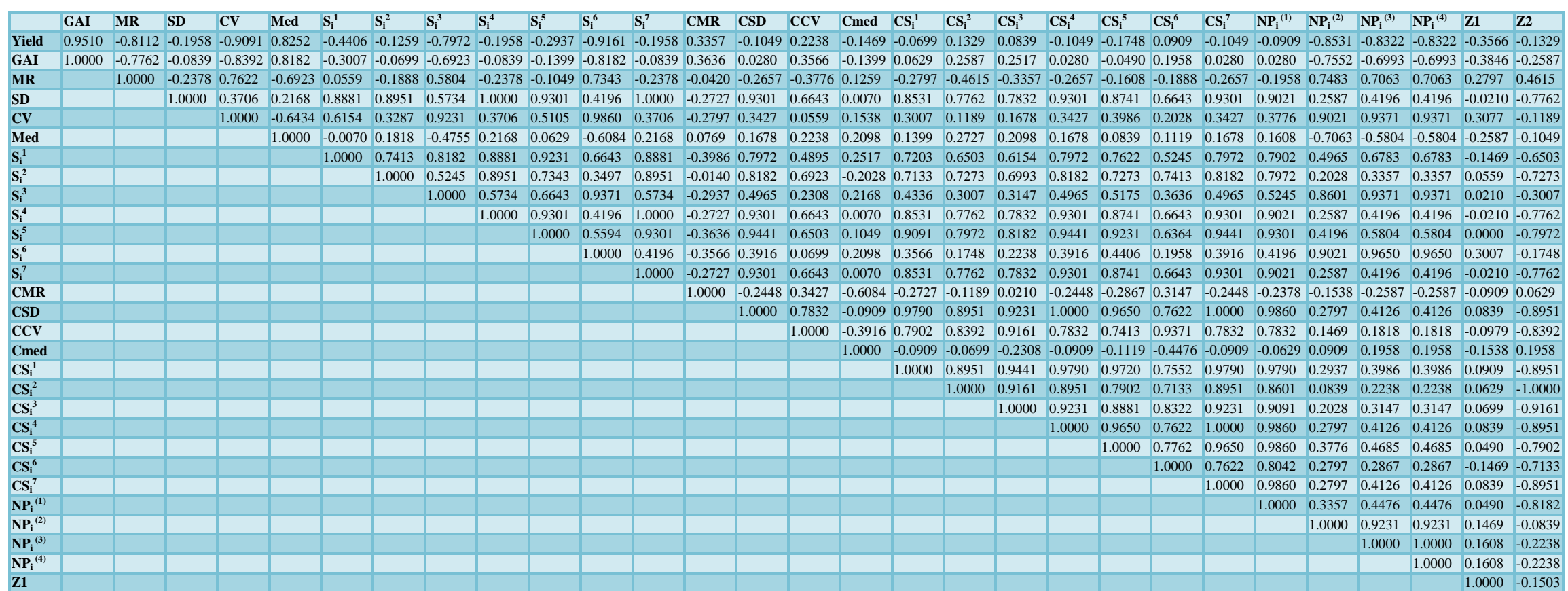


Int.J.Curr.Microbiol.App.Sci (2020) 9(7): 1167-1190

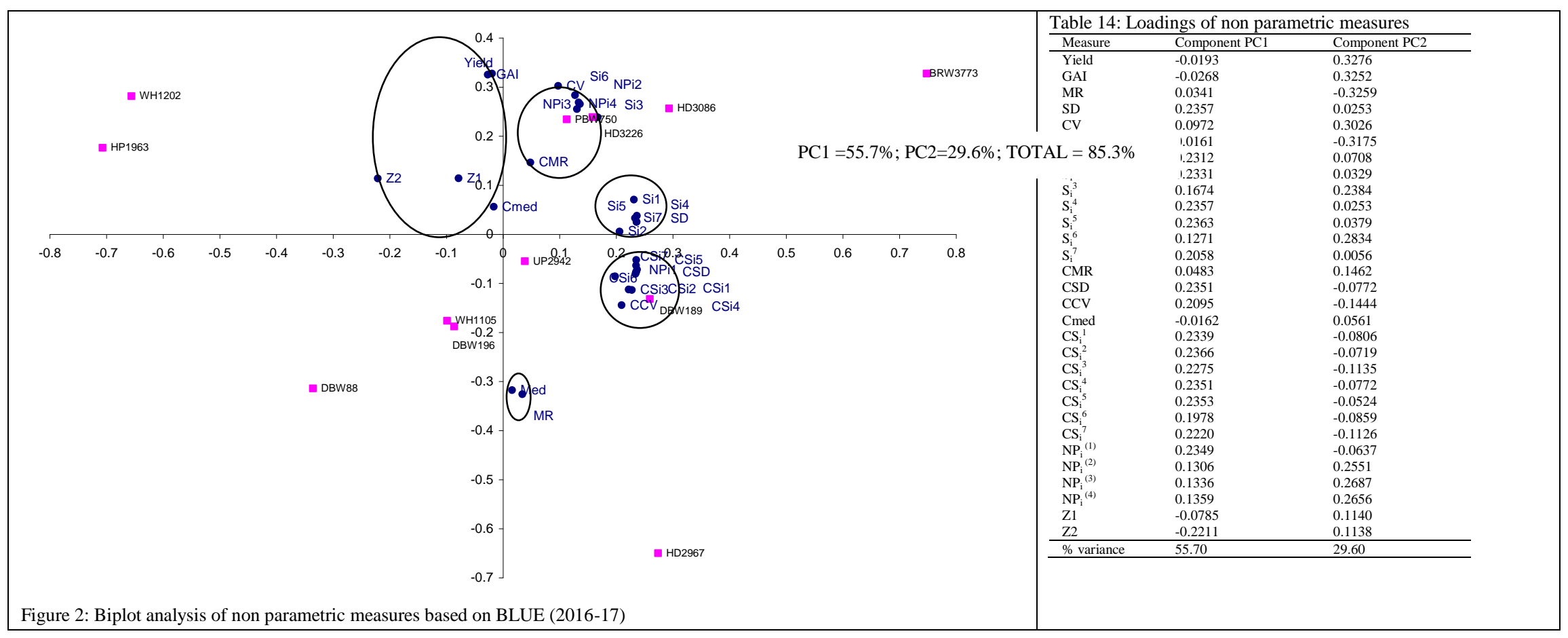


Table.15 Spearman's rank correlation values among non parametric measures (2017-18) BLUP

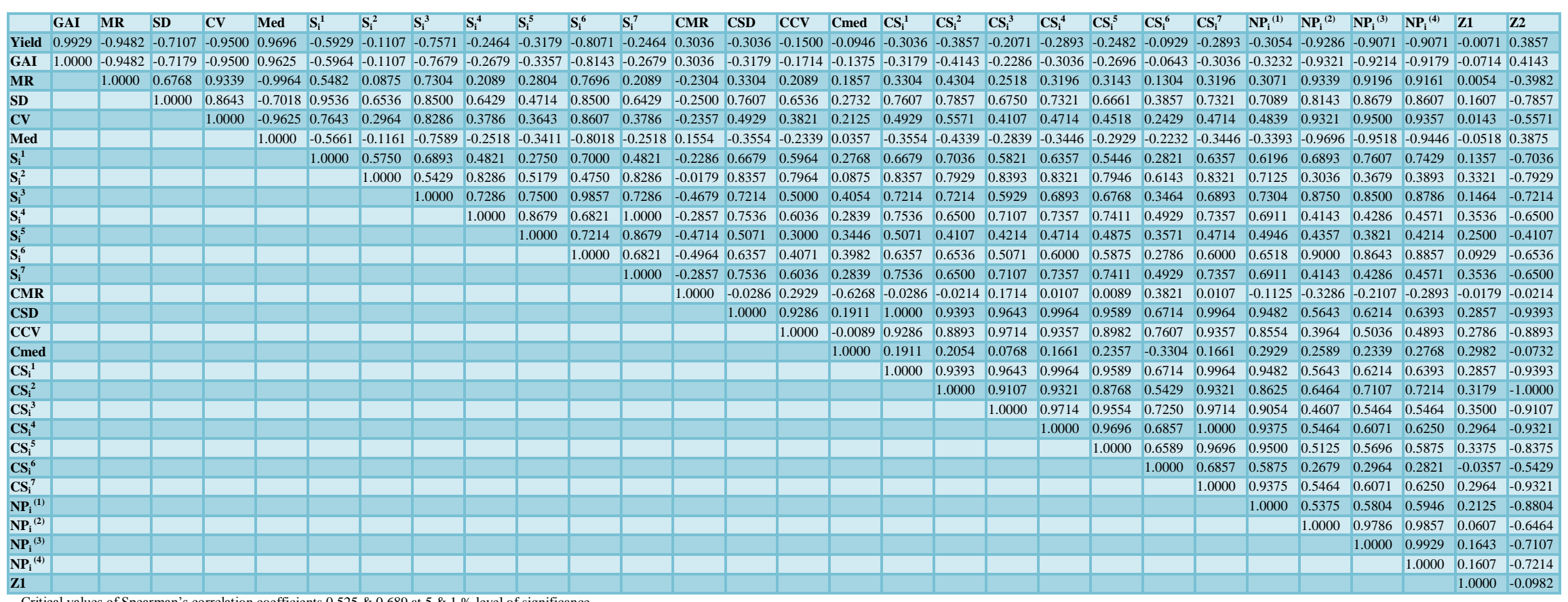




\section{Int.J.Curr.Microbiol.App.Sci (2020) 9(7): 1167-1190}

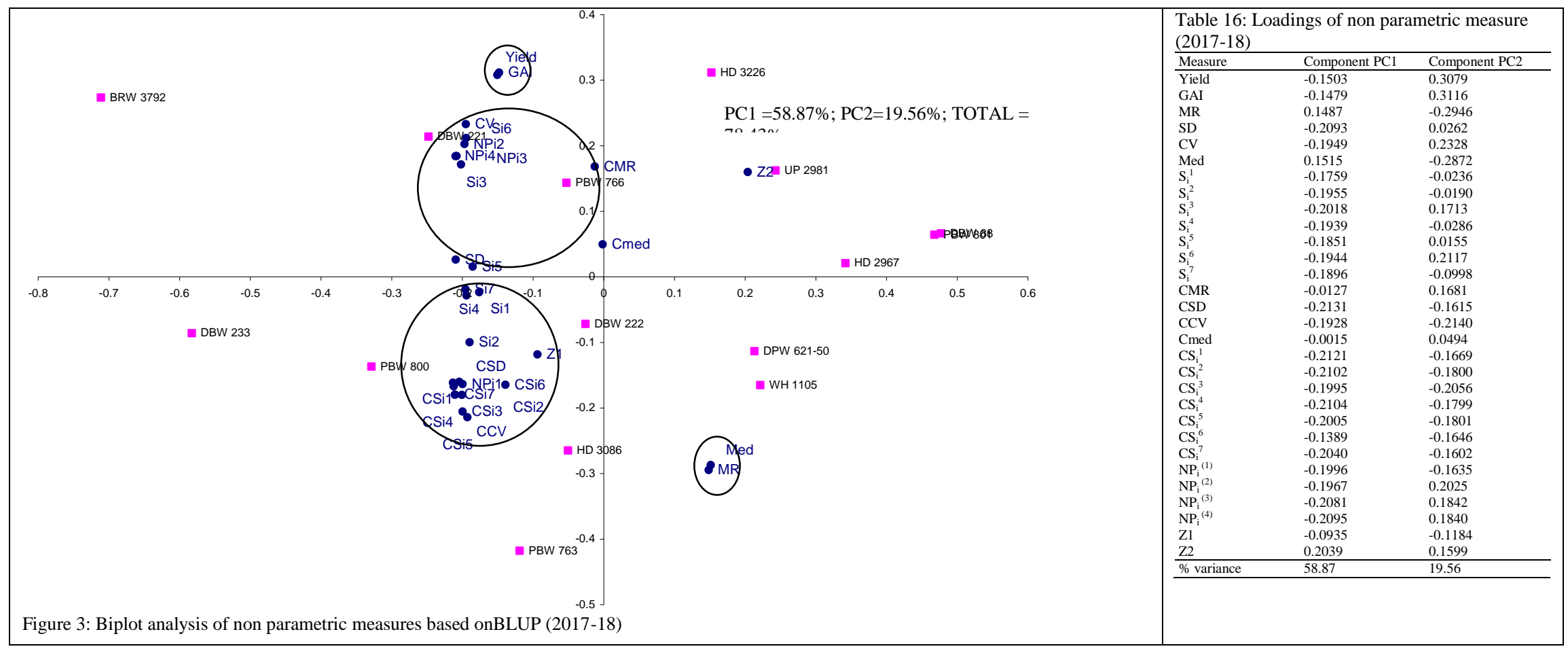


Table.17 Spearman's rank correlation values among non parametric measures (2017-18) BLUE

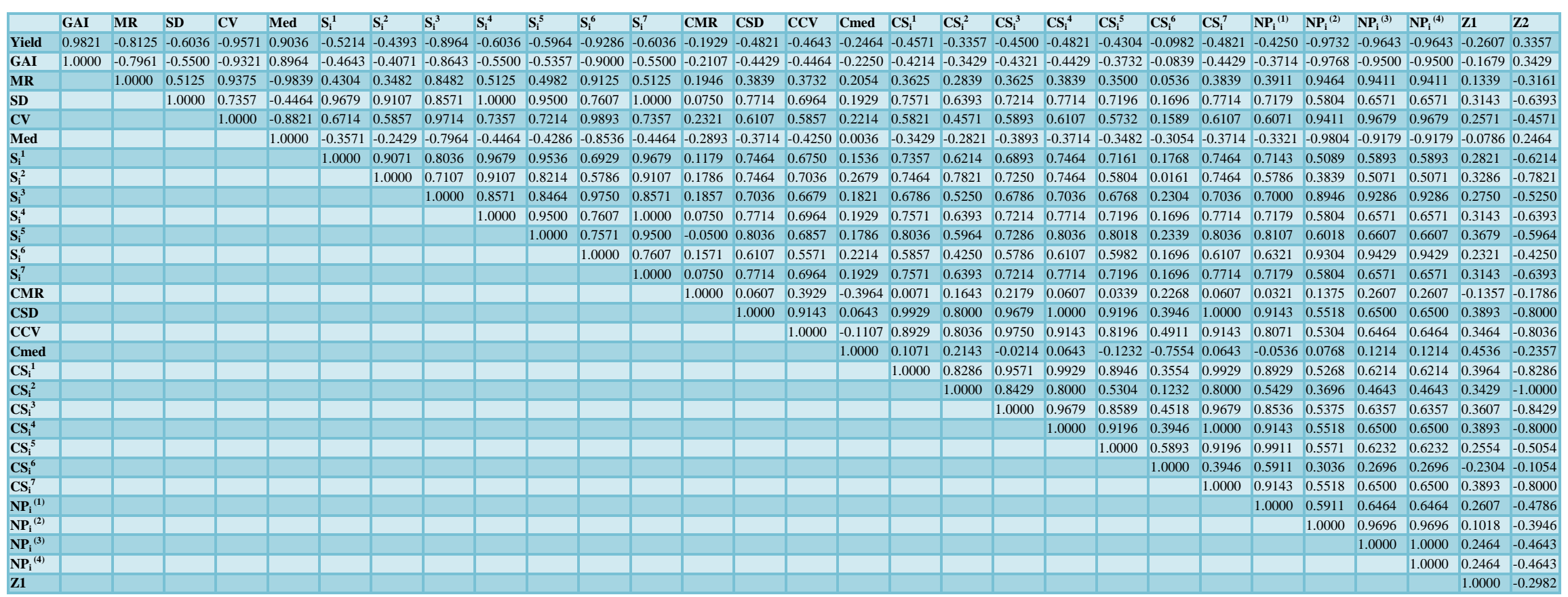


Int.J.Curr.Microbiol.App.Sci (2020) 9(7): 1167-1190

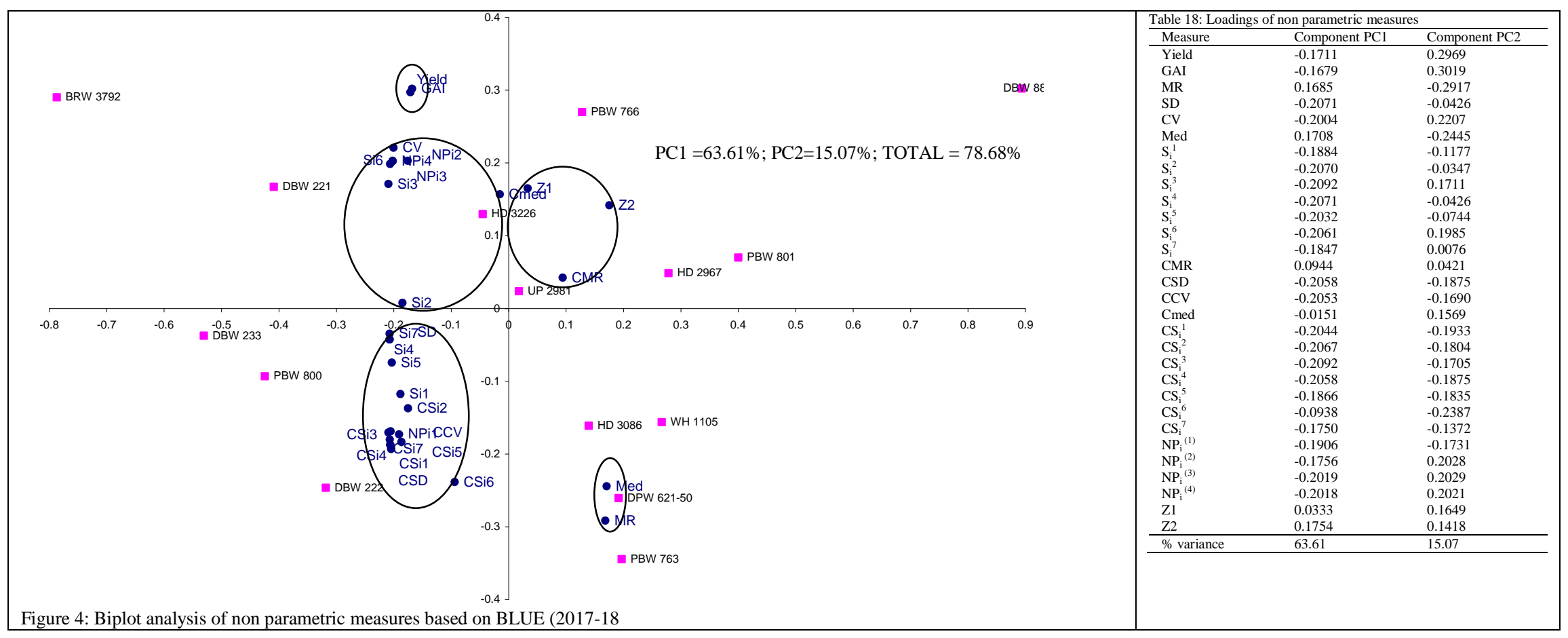




\section{Biplot graphical analysis}

Loadings of the first two principal components axes (PCA) of ranks of nonparametric measures were shown in table 16 . Both significant PAC's accounting for $78.4 \%$ of the variations in the variables. The PCA1 versus PCA2 were used to generate the clustering pattern of measures by biplot as illustrated in Fig. 3. Biplot analysis expressed both types of large and small clusters of measures. Smaller clusters of only two measures i.e. Yield with GAI and MR with Med are observed in graphical analysis. CV along with CMR expressed affinity with, SD CMed, $\mathrm{NP}_{\mathrm{i}}^{(2)}, \mathrm{NP}_{\mathrm{i}}^{\left({ }^{(3)}\right.}, \mathrm{NP}_{\mathrm{i}}^{(4)}, \mathrm{S}_{\mathrm{i}}{ }^{3}, \mathrm{~S}_{\mathrm{i}}^{5}, \mathrm{~S}_{\mathrm{i}}{ }^{6}$. Large cluster comprises of CCV, CSD, $\mathrm{NP}_{\mathrm{i}}^{(1)}, \mathrm{S}_{\mathrm{i}}{ }^{1}$, $\mathrm{S}_{\mathrm{i}}{ }^{2}, \mathrm{~S}_{\mathrm{i}}{ }^{4}, \mathrm{CS}_{\mathrm{i}}{ }^{1}, \mathrm{CS}_{\mathrm{i}}{ }^{2}, \mathrm{CS}_{\mathrm{i}}{ }^{3}, \mathrm{CS}_{\mathrm{i}}{ }^{4}, \mathrm{CS}_{\mathrm{i}}{ }^{5}, \mathrm{CS}_{\mathrm{i}}{ }^{6}, \mathrm{CS}_{\mathrm{i}}{ }^{7}$ and Z1measures.

\section{Analytic analysis as per BLUE's}

Higher average yield had expressed by G4,G2,G7 wheat genotypes, whereas GAI selected G4, G2, G8 as genotypes of choice, large values of mean ranks selected G5, G14, G15 more over the consistent yield of G9, G14, G6 expressed by least values of standard deviation (Table 9). G14, G5, G11 would be genotypes of least variations as pointed by values of coefficient of variation; $S_{i}{ }^{1}$ measure selected G14, G9, G6 opposed to G9, G14, G6 by $\mathrm{S}_{\mathrm{i}}{ }^{2} \& \mathrm{~S}_{\mathrm{i}}{ }^{3}$ measures. Same set of genotypes G6, G9, G14 considered by $\mathrm{Si}^{4}$ along with $\mathrm{S}_{\mathrm{i}}^{5}$, G14, G5, G11 genotypes favoured $S_{i}^{6}$ whereas $S_{i}^{7}$ pointed towards $G 9$, G14, G6 wheat genotypes.

Average mean of ranks as per corrected yield values selected G14, G5, G1 and corrected standard deviation observed suitability of G14, G13, G9 genotypes. Coefficient of variation as per corrected yield values exhibited G14, G6, G13 while median values for $\mathrm{G} 11, \mathrm{G} 3, \mathrm{G} 9$ and $\mathrm{G} 14, \mathrm{G} 13, \mathrm{G} 9$ by $\mathrm{CS}_{\mathrm{i}}{ }^{1}$, G14, G6, G15 by $\mathrm{CS}_{\mathrm{i}}{ }^{2}, \mathrm{CS}_{\mathrm{i}}^{3}$ pointed for G14, G6,G13 \& as per $\mathrm{CS}_{\mathrm{i}}{ }^{4}, \mathrm{CS}_{\mathrm{i}}{ }^{5} \& \mathrm{CS}_{\mathrm{i}}{ }^{7}$ values
G14,G13,G9 while G14,G13, G1 by $\mathrm{CS}_{\mathrm{i}}^{6}$ (table 10). No parametric measures while considering ranks of genotypes by original and corrected yield values $\mathrm{NP}_{\mathrm{i}}{ }^{(1)}$, (G14,G9,G13); $\mathrm{NP}_{\mathrm{i}}^{(2)}(\mathrm{G} 14, \mathrm{G} 5, \mathrm{G} 13), \mathrm{NP}_{\mathrm{i}}^{\left({ }^{(3)}\right.}$ $\& \mathrm{NP}_{\mathrm{i}}{ }^{(4)}(\mathrm{G} 14, \mathrm{G} 5, \mathrm{G} 15)$, also selected G4, G5, G8 wheat genotypes and G2 along with G7 would be of unsuitable type as per value of $\mathrm{NP}_{\mathrm{i}}{ }^{(1)}, \mathrm{NP}_{\mathrm{i}}{ }^{(2)}, \mathrm{NP}_{\mathrm{i}}{ }^{(3)}, \mathrm{NP}_{\mathrm{i}}{ }^{(4)}$ whereas values of measures Z1 observed G15, G12, G8 and G8, G12, G15 by Z2 values.

Value of the $\chi^{2}$ statistic was less than table of $\chi^{2}(0.05,290)=124.3(135.8)$, which resulted an overall similarity among non-parametric measures. Value of $\mathrm{Z}_{1}$ sum $=11.39$ and $\mathrm{Z}_{2}$ sum $=11.28($ Table 10) were less than the critical value of $\chi^{2}(0.05,29)=42.6$. This indicated the non-significant differences among genotypes as per ranks of $\mathrm{CS}_{\mathrm{i}}{ }^{1}$ and $\mathrm{CS}_{\mathrm{i}}{ }^{2}$ measures. Unstable performance of DBW 88 judged by larger values as compared to the critical value of $\chi^{2}(0.05,1)=3.84$.

\section{Association analysis}

Yield has expressed highly significant positive correlation with GAI, Med and negative correlation with $\mathrm{MR}, \mathrm{CV}, \mathrm{S}_{\mathrm{i}}{ }^{3}$, $\mathrm{S}_{\mathrm{i}}^{6}, \mathrm{NP}_{\mathrm{i}}{ }^{(2)}, \quad \mathrm{NP}_{\mathrm{i}}^{(3)}, \quad \mathrm{NP}_{\mathrm{i}}^{(4)}$ measures as Spearman's rank correlation values among non-parametric measures had put in table 17. GAI measure showed negative correlations with MR, $\mathrm{CV}, \mathrm{S}_{\mathrm{i}}{ }^{3}, \mathrm{~S}_{\mathrm{i}}{ }^{6}, \mathrm{NP}_{\mathrm{i}}^{(2)}, \mathrm{NP}_{\mathrm{i}}{ }^{(3)}, \mathrm{NP}_{\mathrm{i}}{ }^{(4)}$ and positive with median. Mean rank of original values (MR) expressed positive correlation with $\mathrm{CV}, \mathrm{S}_{\mathrm{i}}{ }^{3}, \mathrm{~S}_{\mathrm{i}}{ }^{6}, \mathrm{NP}_{\mathrm{i}}^{(2)}, \mathrm{NP}_{\mathrm{i}}^{(3)}, \mathrm{NP}_{\mathrm{i}}^{(4)}$ and negative with Med. Measure SD maintained highly significant and significant positive correlations with almost all the measures exception of Z2. Measure CV also showed significant positive correlation with nearly all measures with negative of Median. More over Median managed negative correlations considered measures. All measures $\mathrm{S}_{\mathrm{i}}{ }^{1}, \mathrm{~S}_{\mathrm{i}}{ }^{2}$, $\mathrm{S}_{\mathrm{i}}{ }^{3}, \mathrm{~S}_{\mathrm{i}}{ }^{4}, \mathrm{~S}_{\mathrm{i}}{ }^{5}, \mathrm{~S}_{\mathrm{i}}{ }^{6}, \mathrm{~S}_{\mathrm{i}}{ }^{7}$ exhibitedindirect relations with $\mathrm{Z} 2$ otherwise only positive correlation 
values were seen with other measures. Mean of ranks based on corrected yield values (CMR) maintained weak relationships of both kinds with most of the measures. CSD, CMR $\&$ CMed had significant positive relationships with all measures whereas negative observed with Z2. Measures based on ranks of corrected yield values $\left(\mathrm{CS}_{\mathrm{i}}{ }^{1}, \mathrm{CS}_{\mathrm{i}}{ }^{2}\right.$, $\mathrm{CSi}^{3}, \mathrm{CS}_{\mathrm{i}}{ }^{4}, \mathrm{CS}_{\mathrm{i}}{ }^{5}, \mathrm{CS}_{\mathrm{i}}{ }^{6}, \mathrm{CS}_{\mathrm{i}}{ }^{7)}$ able to maintain positive relationships with non parametric measures with highly significant negative values of correlation with $\mathrm{Z} 2$ values. Four non-parametric measures based of adjusted ranks of genotypes within each test environment $\mathrm{NP}_{\mathrm{i}}^{(1)}, \mathrm{NP}_{\mathrm{i}}^{(2)}, \mathrm{NP}_{\mathrm{i}}^{(3)}, \mathrm{NP}_{\mathrm{i}}^{(4)}$ had positive relationships with others as well as themselves while expressed negative relationships with $\mathrm{Z} 2$. Lastly $\mathrm{Z} 1$ is related to $\mathrm{Z} 2$ in inverse manner.

\section{Biplot graphical analysis}

Table 18 showed the loadings of the first two PCA of non parametric measures, as contributed about for $78.7 \%$ of the variations of measures. The PCA1 versus PCA 2 were used to generate possible clusters in the biplot analysis as illustrated in Figure 4.Two small clusters of Med with MR and other of Yield with GAI also observed for this data set of genotypes evaluated with major locations of this zone of the country. CMR joined hands with $\mathrm{Z} 1$ and $\mathrm{Z} 2$ to form a cluster of three measures. $\mathrm{CV}$ with $\mathrm{CMed}$ and $\mathrm{NP}_{\mathrm{i}}^{(2)}, \mathrm{NP}_{\mathrm{i}}^{(3)}$, $\mathrm{NP}_{\mathrm{i}}{ }^{(4)}$ as well $\mathrm{S}_{\mathrm{i}}{ }^{2}, \mathrm{~S}_{\mathrm{i}}{ }^{3}, \mathrm{~S}_{\mathrm{i}}{ }^{6}$ comprised in cluster.Largest cluster consists of SD, CSD, $\mathrm{CCV}, \mathrm{NP}_{\mathrm{i}}{ }^{(1)}, \mathrm{S}_{\mathrm{i}}{ }^{1}, \mathrm{~S}_{\mathrm{i}}{ }^{4}, \mathrm{~S}_{\mathrm{i}}{ }^{5}, \mathrm{~S}_{\mathrm{i}}{ }^{7}, \mathrm{CS}_{\mathrm{i}}{ }^{1}, \mathrm{CS}_{\mathrm{i}}{ }^{2}, \mathrm{CS}_{\mathrm{i}}{ }^{3}$ $, \mathrm{CS}_{\mathrm{i}}{ }^{4}, \mathrm{CS}_{\mathrm{i}}{ }^{5}, \mathrm{CS}_{\mathrm{i}}{ }^{6}, \mathrm{CS}_{\mathrm{i}}{ }^{7}$ measures.

BLUP's of wheat genotypes provide more valid estimates of yield in multi environment trials and more variations accounted by first two significant principal components of nonparametric measures. More affinity among measures had reflected by a smaller number of clusters in biplot analysis based on
BLUP's. Association of $\mathrm{S}_{\mathrm{i}}{ }^{1}, \mathrm{CSi}^{1}, \mathrm{NPi}^{(1)}$ with other measures is independent of ranks as per BLUP or BLUE of genotypes. Positive and direct relationships exhibited by these measures with other non-parametric measures.

\section{Acknowledgements}

Authors sincerely acknowledge the training by Dr J Crossa and financial support by Dr. A.K Joshi \& Dr RP Singh CIMMYT, Mexico along with hard work of the staff at coordinating centers of AICW\&BIP project to carry out the field evaluation and data recording.

\section{Conflict of Interest}

No conflict of interest among authors for this study.

\section{References}

Ahmadi, Jafar\&Vaezi, Behrouz \&Shaabani, Akbar \&Khademi, Karim \&Ourang, Sedigheh\& Pour-Aboughadareh, Alireza. (2015). Non-parametric Measures for Yield Stability in Grass Pea (Lathyrus sativus L.) Advanced Lines in Semi Warm Regions. Journal of Agricultural Science and Technology. 17:1825-1838.

Balalić, I., Zorić M., Miklič V., Dušanić N., Terzić S. and Radić V. (2011). Nonparametric stability analysis of sunflower oil yield trials. Helia 34: 67-77.

Delić N., Stanković G. and KonstatinovK .(2009). Use of non parametric statistics in estimation of genotypes stability. Maydica 54: 155-160.

Farshadfar E., Mahmudi N. and SheibaniradA .(2014). Nonparametric methods for interpreting genotype $\times$ environment interaction in bread wheat genotypes. Journal of Biodiversity \& Environmental Sciences 4: 55-62.

Huehn M. (1990a). Non-parametric measures of phenotypic stability. Part 1: Theory. Euphytica 47:189-194. 
Huehn M. (1990b). Non-parametric measures of phenotypic stability: Part 2. Application. Euphytica 47:195-201

Hühn M. and Leon J. (1995). Nonparametric analysis of cultivar performance trials: experimental results and comparison of different procedures based on ranks. Agronomy Journal 87:627-632.

Karimizadeh R., Mohammadi M., Sabaghnia N. and Shefazadeh M.K. (2012). Using Huehn's nonparametric stability statistics to Investigate Genotype $\times$ Environment interaction. Notulae Botanicae Horti Agrobotanici Cluj-Napoca 40: 293-301

Khalili, M., and A. Pour-Aboughadareh. (2016). Parametric and non- parametric measures for evaluating yield stability and adaptability in barley doubled haploid lines. Journal of Agricultural Science and Technology 18: 789-803

Kilic, H., M. Akcura and H. Aktaş. (2010). Assessment of parametric and nonparametric methods for selecting stable and adapted durum wheat genotypes in multienvironments. Not. Bot. Horti. Agrobo., 38, 271-279.

Mahtabi E., Farshadfar E. and Jowkar M.M. (2013). Non parametric estimation of phenotypic stability in chickpea (Cicer arietinum L.). International Journal of Agriculture and Crop Science 5: 888-895.

Mohammadi R., Farshadfarar E. and Amri A .(2016). Comparison of rank-based stability statistics for grain yield in rainfed durum wheat. New Zealand Journal of Crop \& Horticulture Science 44: 25-40.

Mortazavian S. M. M. and Azizinia S. (2014). Nonparametric stability analysis in multienvironment trial of canola. Turkish Journal Field Crops 19(1): 108-117.

Nassar R. and Huehn M. (1987). Studies on estimation of phenotypic stability: tests of significance for non-parametric measures of phenotypic stability. Biometric 43: 45- 53.

Piepho H.P. and Lotito S. (1992). Rank correlation among parametric and nonparametric measures of phenotypic stability. Euphytica 64: 221-225.

Pour-Aboughadareh, A., M. Yousefian, $\mathrm{H}$. Moradkhani, P. Poczai, and K. H. M. Siddique. (2019). STABILITYSOFT: A new online program to calculate parametric and non- parametric stability statistics for crop traits. Applications in Plant Sciences 7(1): e1211

Rasoli, V., Farshadfar E. and Ahmadi J. (2015). Evaluation of Genotype $\times$ Environment Interaction of grapevine genotypes (Vitis vinifera L.) by non parametric method. Journal of Agricultural Science and Technology, 17: 1279-1289.

Sabaghnia N., Karimizadeh R. and Mohammadi M. (2012). The use of corrected and uncorrected nonparametric stability measurements in Durum wheat multienvironmental Trials. Spanish Journal of Agricultural Research 10: 722-730

Thennarasu K. (1995). On certain non-parametric procedures for studying genotypeenvironment interactions and yield stability. Unpublished Ph.D. Thesis. P.G. School, IARI, New Delhi

Vaezi, B., A. Pour-Aboughadareh, A. Mehraban, T. Hossein-Pour, R. Mohammadi, M. Armion, and M. Dorri. (2018). The use of parametric and non- parametric measures for selecting stable and adapted barley lines. Archives of Agronomy and Soil Science 64: 597-611

Zali H., Farshadfar E. and Sabaghpour S. H. (2011). Non-parametric analysis of phenotypic Stability in chickpea (Cicer arietinum L.) genotypes in Iran. Crop Breeding Journal, 1(1): 89-100.

\section{How to cite this article:}

Ajay Verma and Singh, G.P. 2020. Non parametric Measures of Stability Compared as per BLUP and BLUE of Wheat Genotypes Evaluated in North Western Plains Zone of the Country. Int.J.Curr.Microbiol.App.Sci. 9(07): 1167-1190. doi: https://doi.org/10.20546/ijcmas.2020.907.136 